\title{
Title: Persistence of SARS-CoV-2 immunity, Omicron's footprints, and projections of epidemic resurgences in South African population cohorts.
}

Authors: Kaiyuan Sun $^{1 *}$, Stefano Tempia ${ }^{2,3,4}$, Jackie Kleynhans ${ }^{2,3}$, Anne von Gottberg ${ }^{2,5}$, Meredith L McMorrow ${ }^{4}$, Nicole Wolter ${ }^{2,5}$, Jinal N. Bhiman ${ }^{2,5}$, Jocelyn Moyes ${ }^{2,3}$, Mignon du Plessis $^{2,5}$, Maimuna Carrim²,5, Amelia Buys ${ }^{2}$, Neil A Martinson ${ }^{6,7}$, Kathleen Kahn ${ }^{8}$, Stephen Tollman $^{8}$, Limakatso Lebina ${ }^{6}$, Floidy Wafawanaka ${ }^{8}$, Jacques du Toit ${ }^{8}$, Francesc Xavier GómezOlivé $^{8}$, Thulisa Mkhencele ${ }^{2}$, Cécile Viboud ${ }^{1 \dagger}$, Cheryl Cohen ${ }^{2,3 \dagger^{*}}$, for the PHIRST group

\section{Affiliations:}

${ }^{1}$ Division of International Epidemiology and Population Studies, Fogarty International Center, National Institutes of Health, Bethesda, Maryland, United States of America.

${ }^{2}$ Centre for Respiratory Diseases and Meningitis, National Institute for Communicable Diseases of the National Health Laboratory Service, Johannesburg, South Africa.

${ }^{3}$ School of Public Health, Faculty of Health Sciences, University of the Witwatersrand, Johannesburg, South Africa.

${ }^{4}$ Influenza Division, Centers for Disease Control and Prevention, Atlanta, Georgia, United States of America.

${ }^{5}$ School of Pathology, Faculty of Health Sciences, University of the Witwatersrand, Johannesburg, South Africa.

${ }^{6}$ Perinatal HIV Research Unit, University of the Witwatersrand, South Africa.

${ }^{7}$ Johns Hopkins University Center for TB Research, Baltimore, Maryland, United States of America.

${ }^{8} \mathrm{MRC} /$ Wits Rural Public Health and Health Transitions Research Unit (Agincourt), School of Public Health, Faculty of Health Sciences, University of the Witwatersrand, Johannesburg, South Africa.

*Corresponding author. Email: kaiyuan.sun@nih.gov (KS); cherylc@nicd.ac.za (CC)

$\uparrow$ These are co-senior authors of this work.

Abstract: Understanding the build-up of immunity with successive SARS-CoV-2 variants and the epidemiological conditions that favor rapidly expanding epidemics will facilitate future pandemic control. High-resolution infection and serology data from longitudinal household cohorts in South Africa reveal high cumulative infection rates and durable cross-protective immunity conferred by prior infection in the pre-Omicron era. Building on the cohort's history of past exposures to different SARS-CoV-2 variants and vaccination, we use mathematical models to explore the 
fitness advantage of the Omicron variant and its epidemic trajectory. Modelling suggests the Omicron wave infected a large fraction of the population, leaving a complex landscape of population immunity primed and boosted with antigenically distinct variants. Future SARS-CoV2 resurgences are likely under a range of scenarios of viral characteristics, population contacts, and residual cross-protection.

One Sentence Summary: Closely monitored population in South Africa reveal high cumulative infection rates and durable protection by prior infection against pre-Omicron variants. Modelling indicates that a large fraction of the population has been infected with Omicron; yet epidemic resurgences are plausible under a wide range of epidemiologic scenarios.

Main Text: A key questions for the long-term control of SARS-CoV-2 is how sequential exposures to different variants of the virus shape population immunity and thereby modulate subsequent epidemic cycles and disease burden. Few studies have characterized the protection conferred by infection over long time periods, particularly in low and middle-income settings where vaccine access is limited and high SARS-CoV-2 infection rates have been reported (1-4). Here we used unique data from two prospectively followed cohorts in South Africa to estimate the strength of cross-protective immunity conferred by infection with successive SARS-CoV-2 variants. We relied on the cohort data to reconstruct the landscape of population immunity prior to the emergence of the Omicron variant, and modeled the trajectory, scale and long-term consequences of the Omicron epidemic in this population.

South Africa experienced three distinct SARS-CoV-2 epidemic waves prior to the emergence of the Omicron variant, with the first wave (June 2020 - December 2020) dominated by the ancestral SARS-CoV-2 strain carrying the D614G mutation (refer to as D614G hereafter) (5), the second wave (December 2020 - May 2021) dominated by the Beta (B.1.351) variant (6), and the 25 third wave (May 2021 - October 2021) dominated by the Delta (B.1.617.2) variant (7). In South Africa, the Omicron variant was first identified in the province of Gauteng Province in November 2021, and swiftly spread nationally and globally, causing rapid growth in case counts relative to prior waves $(7,8)$. Similar patterns of rapid growth despite high levels of pre-existing immunity from infection and vaccination have also been reported in numerous countries across the world, 
with Omicron replacing Delta in multiple global locations, even when prevalence of Delta was $\operatorname{high}(9,10)$.

The apparent fitness advantage of the Omicron variant over Delta could be driven by immune evasion, increased intrinsic transmissibility, or a combination of both. The immune evasion hypothesis is supported by an increased reinfection risk coinciding with the rise of the Omicron variant $(7,8,11,12)$. Further support for this hypothesis comes from in vitro analyses of sera from convalescent patients (infected with pre-Omicron variants) and vaccinated individuals, which show reduced neutralization titers against Omicron compared to earlier variants (13-16). Similarly, data from multiple settings have shown decreased vaccine effectiveness against Omicron $(17,18)$. Separately, epidemiological and experimental data point to a reduced clinical severity of Omicron $(17,19)$, possibly due to increased tropism for the upper respiratory tract rather than the lung, which could also promote higher transmission relative to pre-Omicron variants. As the Omicron wave subsides, the relative contribution of these factors to Omicron's spread remains elusive, in part due to uncertainty in the level of population immunity before the rise of Omicron. Further, Omicron's rapid spread poses immense pressure on SARS-CoV-2 testing capabilities, and its relatively benign course in most people make it difficult to assess the full scope of the epidemic.

To understand the long-term dynamics of SARS-CoV-2, we leveraged data from two longitudinal household cohorts followed over a 13-month period, from July 2020 to August 2021 in rural and urban areas of South Africa (Prospective Household study of SARS-CoV-2, Influenza and Respiratory Syncytial virus community burden, Transmission dynamics and viral interaction in South Africa, PHIRST-C, where "C" stands for COVID-19, previously described in (4)). We relied on the results of densely sampled respiratory and serologic specimens testing from 222 households to model the kinetics of viral shedding, transmission dynamics among household members, and cross-protection between successive variants circulating prior to the emergence of Omicron. We used population-level models calibrated against data from these prospective cohorts and surveillance efforts to clarify long-term patterns of immunity acquisition, the impact of 
immune evasion, and future epidemic trajectories for SARS-CoV-2 in the aftermath of the Omicron wave.

\section{Overview of SARS-CoV-2 epidemiology in study sites.}

The PHIRST-C cohort captured the dynamics of three waves of SARS-CoV-2 infections in rural and urban site located in two provinces of South Africa (see methods for details). In total, 1,200 individuals living in 222 randomly selected households were enrolled and followed up twice a week for SARS-CoV-2 real-time reverse transcription polymerase chain reaction (rRT-PCR) testing and symptom monitoring, while blood draws were obtained every 2 months for SARSCoV-2 serologic tests. Throughout the study, we used a broad measurement of prior and ongoing infections including both serologic and virologic evidence, irrespective of symptoms. In Figure 1A-B, we show the weekly SARS-CoV-2 epidemic curves in the district where each study site was located (20). Population vaccination started in June 2021 in South Africa and the fraction of the cohort population fully vaccinated remained below $10 \%$ at the conclusion of the study in September 2021 (Figure 1A-B). We did not evaluate vaccine effectiveness in this study and focused on protection conferred by prior SARS-CoV-2 infection. However, we consider the impact of vaccination in projections of the Omicron wave and post-Omicron future.

In the rural site, baseline enrollment visits started prior to the peak of the first epidemic wave. The seroprevalence of anti-SARS-CoV-2 nucleocapsid antibodies was 1.1\% (5/445) at enrollment, increased to $7.3 \%(42 / 574)$ after the first wave (third blood draw), 25.4\% (151/595) after the second wave (fifth blood draw), and reached 39.1\% (227/581) around the peak of the third wave (seventh blood draw). The timing and individual results of the serological assay are visualized in Figure 1A. During the study period (July 2020 to August 2021), 50.9\% (327/643) of individuals tested positive by rRT-PCR for at least one infection episode. The cumulative infection rate (confirmed by either a rRT-PCR or a serological test) was $59.7 \%(384 / 643)$ by the end the study 25 in the rural cohort. In contrast, in the urban site, enrollment started near the time of the peak of the first wave and SARS-CoV-2 seroprevalence was higher at enrollment (14.3\%, 73/511), increased to $27.0 \%$ (143/530) after the first wave, 40.3\% (207/514) after the second wave, and reached 55.7\% $(279 / 501)$ around the peak of the third wave (see Figure 1B for bi-monthly results). During the study period, $53.1 \%(296 / 557)$ of participants tested positive by rRT-PCR for at least one infection 
episode. The cumulative infection rate (confirmed by either a rRT-PCR or a serological test) was $69.4 \%(387 / 557)$ in the urban cohort by the end of the study.

In total across both sites, we observed 669 rRT-PCR-confirmed infection episodes, including 599/669 (89.5\%) primary infections and 70/669 (9.5\%) reinfections. Lineage-specific rRT-PCR and sequencing data revealed that $14.3 \%$ (96/669) of infections were D614G, 33.2\% (222/669) were Beta, 44.1\% (295/669) were Delta, 2.7\% were other lineages, and 5.7\% (38/669) were inconclusive. Figure $1 \mathrm{E}-\mathrm{F}$ shows the relative prevalence of different lineages over time for the rural and urban site, respectively (details in Materials and Methods Section 3.2).

\section{Kinetics of viral RNA shedding.}

To study the risk of infection and re-infection in the cohort, and better understand acquisition of immunity before the rise of Omicron, we first built a time-varying model that captured the dynamics of viral RNA shedding for each individual in the cohort, adjusted for host characteristics and variant types. Household exposure depends on the level of viral shedding among household members; to obtain a correlate of shedding intensity, we used the serial $\mathrm{Ct}$ values of nasal swab specimens collected twice-weekly and tested for SARS-CoV-2 using rRT-PCR. We considered the difference between the Ct value of the rRT-PCR test of a specimen and the Ct value of the detection limit of our rRT-PCR assay as a proxy for the RNA shedding intensity (refer to as $d C t$ hereafter). We used the serial rRT-PCR test results to model the shedding kinetics of SARS-CoV2 infection episodes, as have others $(22,23)$. To account for the potential role of adaptive immunity in limiting transmission in the later phases of infection, we allowed for different transmission risks during the viral RNA proliferation stage (before peak shedding) and the viral RNA clearance stage (after peak shedding), with shedding increases and decreases assumed to following linear curves on the scale of $\mathrm{Ct}$ values. Because the nasal swab sampling period ended on August 28, 2021, around the peak of the Delta wave in both sites, we limit our analysis to infection episodes with first positive PCR specimen 30 days prior to the end of sampling to avoid censoring bias.

Figure 2 A-C shows the RNA shedding kinetics of the D614G, Beta, and Delta variants respectively. All three variants had similar shedding kinetics characterized by a short proliferation stage (Figure 2E, median and interquartile range (IQR) for D614G: 2.8 (1.2 - 4.0) days, Beta: 2.7 $(1.6$ - 3.4) days, Delta: 3.0 (1.9 - 3.7) days) and a longer clearance stage (Figure 2F, median and 
IQR for D614G: 7.1 (3.0 - 10.9) days, Beta: 7.4 (3.9 - 10.3) days, Delta: 7.9 (3.9 - 10.8) days). After adjusting for age, sex, body mass index (BMI), and HIV infection status, symptomatic infections had significantly higher peak $d C t$ value (peak shedding intensity) than asymptomatic infections (Figure 2H). Beta variant's peak $d C t$ value was higher than D614G, while Delta's was the highest among the three (Figure $2 \mathrm{H}$ ). In addition, individuals infected with the Delta variant shed RNA for significantly longer than D614G (Figure 2I). We found that prior infection significantly reduced peak shedding by $3.7 \mathrm{Ct}(95 \% \mathrm{CI} 2.0-5.5)$ and shedding duration by 3.6 days (95\% CI $2.0-5.2)$ upon reinfection (Figure $2 \mathrm{H}-\mathrm{I})$.

The population of the PHIRST-C cohort had a high prevalence of HIV, $13 \%$ in the rural site and $16 \%$ in the urban site, reflecting the burden of HIV infections in South Africa. However, in this cohort, most (93.8\%) persons living with HIV (PLWH) had CD4+ T cell counts $\geq 200$ cells/ml, and they did not differ from HIV-uninfected individuals in terms of SARS-CoV-2 shedding (Figure $2 \mathrm{H}-\mathrm{I}$ ). We found that individuals with low CD4+ T cell counts $(<200$ cells $/ \mathrm{ml})$ tended to shed for longer and with higher peak $d C t$ when compared to HIV-uninfected individuals but there was limited statistical power to detect these differences (Figure $2 \mathrm{H}-\mathrm{I}$ ).

\section{Infection risk and protection against reinfection.}

Reconstruction of the variant-specific shedding kinetics of each infected individual allowed us not only to infer the timing of their infections, but also to evaluate the daily intensity of exposure to SARS-CoV-2 within their households. We used logistic regression to explore how variant type and prior infection history affect the risk of SARS-CoV-2 infection and reinfection, after adjustment for SARS-CoV-2 exposure risk and host factors. We regressed the daily infection risk of each individual on variant type, time since prior SARS-CoV-2 infection, age (allowing for variant-specific age patterns), sex, BMI, HIV infection status, household size, crowding, study site, household exposures to SARS-CoV-2, and community SARS-CoV-2 infection prevalence within the cohort. We found that the risk of acquiring infection increased with the sum of the $d C t$ s of infected household members, with a stronger effect in the proliferation than the clearance phase (Figure 2J). One-unit increase in the sum of $d C t$ s among household members in the proliferation stage led to a $114 \%(95 \%$ CI $79 \%$ - 155\%) increase in the odds of infection whereas one unit increase in the clearance stage led to $60 \%(95 \%$ CI $40 \%-84 \%)$ increase in the odds of infection. 
medRxiv preprint doi: https://doi.org/10.1101/2022.02.11.22270854; this version posted February 13, 2022. The copyright holder for this preprint (which was not certified by peer review) is the author/funder, who has granted medRxiv a license to display the preprint in perpetuity. It is made available under a CC-BY-NC-ND 4.0 International license .

Compared to D614G, we found that infectiousness was highest for the Delta variant, followed by the Beta variant, after adjusting for household exposures and community prevalence, among other risk factors (odds ratio against D614G: Delta 2.17, 95\% CI 1.44 - 3.28, Beta 1.53, 95\% CI 1.06 2.20). The difference between Delta and Beta's infectiousness was not statistically significant, with overlapping confidence intervals in their odds ratios. We found that prior infection provided durable protection against reinfection throughout the study period. Compared to seronegative individuals, prior infection was 93\% (95\% CI 85 - 96\%) protective against reinfection for the first 3 months and decreased marginally to $87 \%$ (95\% CI $79-93 \%)$ after 9 months (Figure 2J). Individuals older than 65 years were significantly less affected during the D614G wave while children and adolescents aged $6-18$ years were significantly more affected during the Delta wave (Figure 2J). In addition, higher BMI and residing in an urban setting were independently associated with increased risk of SARS-CoV-2 infection. We found that individuals with low CD4 cell counts $(<200)$ tended to be more susceptible to acquisition of SARS-CoV-2 when compared to HIVuninfected individuals, but there was limited statistical power to detect this difference (Figure 2J). Finally, we did not find significant associations between SARS-CoV-2 infection risk and sex, household size or crowding (Figure 2J)

\section{Projecting the Omicron Wave and post-Omicron futures in PHIRST-C's urban site.}

The study cohorts provide estimates of the duration and degree of cross-protective immunity between SARS-CoV-2 variants predating Omicron, with evidence of persistence of clinical protection beyond a year for these variants. Armed with these estimates, we used mathematical models to explore a range of plausible scenarios compatible with the observed transmission dynamics of the Omicron epidemic wave in South Africa and project post-Omicron futures. Specifically, we explore how Omicron's potential differences (relative to Delta) in infectivity, immune evasion, and severity could shape the scale and severity of the Omicron epidemic wave and the likelihood of recurrences of SARS-CoV-2 outbreaks post-Omicron. We focused the analysis on the study's urban cohort, which is sampled from the city of Klerksdorp in Dr Kenneth Kaunda Health District located on a national transport route, and the population is well-mixed with the district population.

Details of the transmission model and calibration procedure can be found in Materials and 
level SARS-CoV-2 surveillance information to reconstruct SARS-CoV-2's antigen exposure(s) history in this population, considering infections and/or vaccinations through time (Figure 3F). We then built a variant-specific transmission model to capture the declining phase of the Delta wave after the end of the PHIRST-C study and infer the population-level transmission rate during that time. Next, we added a second strain to our model to account for Omicron's dynamics, with free parameters representing the relative infectiousness of Omicron vs Delta $R_{0}^{\text {Omicron }} / R_{0}^{\text {Delta }}$, Omicron's degree of immune evasion against infection among individuals infected by prior variants $\sigma_{O m}^{i}$, and Omicron's degree of immune evasion against transmission given reinfection $\sigma_{O m}^{t \mid i}$ (with higher values of $\sigma_{O m}^{i}\left(\sigma_{O m}^{t \mid i}\right)$ indicating higher degrees of immune evasion, where $\sigma_{O m}^{i}\left(\sigma_{O m}^{t \mid i}\right)=0$ corresponds to no evasion while $\sigma_{O m}^{i}\left(\sigma_{O m}^{t \mid i}\right)=1$ indicates $100 \%$ evasion, detailed in Materials and Methods Section 4). We explored the parameter space and initial conditions of Omicron's introduction and selected epidemic trajectories that were compatible with the observed growth advantage of Omicron over Delta and the peak timing of the Omicron wave - the two most reliable epidemiologic observations during the Omicron epidemic. We then evaluated Omicron's possible epidemic trajectories for the remaining parameters conditional on the observed growth rate and peak timing. Lastly, we selected one scenario that was most compatible with independent evidence on the degree of Omicron's immune evasion and projected the likelihood of resurgence of different variants (Figure 3F).

Assuming that the Delta variant was in an exponentially declining phase after week 35 of 2021 and that the Omicron variant was growing exponentially until week 48 of 2021 in the study urban district (20), we estimated that the daily growth rate was -0.063 for Delta and 0.275 for Omicron, which translates into a growth advantage of 0.338 per day for Omicron over Delta (Figure S3). The transmission models of Delta and Omicron were calibrated to match their observed growth rates during this period, respectively. In Figure 3A, we show the trade-off between the estimated ratio of basic reproduction numbers between Omicron and Delta $R_{0}^{\text {Omicron }} / R_{0}^{\text {Delta }}$ and different degrees of evasion of protection against infection $\sigma_{O m}^{i}$ and transmission $\sigma_{O m}^{t \mid i}$. We assumed mean intrinsic generation times of 5 and 4 days for Delta and Omicron (21). We found that across the full range of immune evasion parameters, Omicron had a higher basic reproduction number than Delta. However, there was clear compensation between immune evasion and intrinsic transmissibility: a higher degree of immune evasion would require a lower basic reproduction 
number for the Omicron variant to match the observed epidemic trajectory. We also found that for all parameters explored, the Omicron epidemic led to higher infection rates than prior epidemic waves, with the most optimistic scenario resulting in an infection rate above $40 \%$. Higher degrees of immune evasion resulted in higher infection rates (Figure 3B). Infections were expected to accumulate within a relatively short period of time, with epidemic duration (measured as 4 times the standard deviation of the onset dates of all infections within the epidemic wave) projected to range from $31-37$ days depending on the parameters. Notably, our model projected that a large fraction of Omicron infections ( $>40 \%$ ) would be reinfections or vaccine breakthrough infections, with higher proportions observed for higher immune evasion parameters (Figure 3D). Furthermore, a low rate of clinical cases was projected for Omicron, after controlling for the number of infections due to this variant. The projected infection case ratio (ICR) for Omicron ranged from $0.4 \%$ to $0.9 \%$ (Figure $3 \mathrm{E}$ ), much lower than the ICRs of $3.6 \%, 3.3 \%$, and $9.4 \%$ estimated for the D614G, Beta, and Delta waves (Table S1). In sensitivity analyses (Figure S5), we further explored the impact of Omicron mean generation times ranging from 3-6 days (22).

To estimate the immune footprint of the Omicron wave and project a post-Omicron future, we considered a reference scenario for Omicron's immune evasion characteristics, guided by independent data. We set $\sigma_{O m}^{i}=0.7$ and $\sigma_{O m}^{t \mid i}=0.2$, corresponding to a drop in protection against infection from $88 \%$ for pre-Omicron variants (Fig. 2J) to $47 \%$ for Omicron $(17,23)$, and a drop in protection against transmission from $60 \%(24)$ to $52 \%$, reflecting weak immune evasion on onward transmission (18). Under this scenario, the estimated $R_{0}^{\text {Omicron }} / R_{0}^{\text {Delta }}$ was 2.4 , the infection rate was $69 \%$, the epidemic lasted 32 days, and the fraction of reinfections and vaccine breakthroughs was 68\% (Figure 3A-D, white dot). In Figure 3F, we visualize the observed incidence of reported SARS-CoV-2 cases in all four epidemic waves in the study district and report the reconstructed infection time series and population level history of SARS-CoV-2 antigen exposures. We found variable levels of under-reporting depending on the SARS-Cov-2 variant, with ICR of 3.6\% (95\% CI $3.4-3.8 \%)$ for D614G, 3.3\% (95\%CI 3.0 - 3.6\%) for Beta, 9.4\% (95\%CI 8.7 - 10.2\%) for Delta (Table S1), and 0.5\% for Omicron. Findings for the D614G and Beta wave in agreement with previous findings (25). In the reference scenario (Figure 3E), more than $90 \%$ of the population was projected to have been infected with one or more SARS-CoV-2 variants by the end of the Omicron wave (Figure 3F). In particular, we estimated that $22.5 \%$ of the population would have seen Omicron as their first SARS-CoV-2 exposure, $16.8 \%$ would have been exposed to a pre- 
Omicron variant and this would remain their only SARS-CoV-2 exposure, and $45.7 \%$ would have experienced Omicron reinfections or vaccine breakthrough infections.

Because the Omicron variant is antigenically distinct from all previously circulating variants in South Africa $(14,15,26)$, the level of immunity conferred by Omicron's primary and breakthrough infections/reinfections against itself, other circulating variants, and new variants, are key determinants for the likelihood of SARS-CoV-2 resurgence (27-29). To study the post-Omicron phase, we explored how different exposure histories could confer different levels of protection against infection with homologous and heterologous variants. Accordingly, in our model, the level of protection varied both with the variant that conferred immunity (whether a live variant or the vaccine) and the hypothetical variant circulating in the post-Omicron future. Protection was expressed as a relative risk, compared to naïve individuals (where $R R^{i}=1$ indicates no protection while $R R^{i}=0$ indicates perfect protection). For simplicity, we assumed that protection against transmission remained constant at $60 \%\left(R R^{t \mid i}=0.4 \quad(24)\right)$. We assessed the risk of future recurrences of different SARS-CoV-2 variants after the initial Omicron wave had subsided, assuming possible changes in contacts to account for erosion in adherence to SARS-CoV-2 interventions over time (models detailed in Materials and Methods Section 6).

Even at the contact rate estimated during the Delta wave, the level of population immunity would not be able to prevent a recuring Omicron epidemic unless past Omicron infection conferred high and durable protection (Figure $3 \mathrm{G}$ ). If contact rates increased 100\% relative to current levels, a return of the Omicron variant would likely cause outbreaks irrespective of the protection afforded by prior Omicron infections (outbreak conditions defined as growth rate larger than zero, Figure $3 \mathrm{H})$. A $100 \%$ increase in contacts is plausible given estimated levels of transmission reduction in South Africa at the end of 2021, which reflect the combined effect of population behavior and seasonality (see Materials and Methods, Section 5.5 and Table S3). In contrast, if contact rates were to remain the same as those observed during the Delta wave, the Delta variant would be unlikely to return and cause new outbreaks, across all ranges of Omicron-specific immunity assumptions against Delta (Figure 3I). With 100\% higher contact rates, some scenarios favored a re-emergence of Delta if Omicron did not protect well against Delta (Figure 3J). We also explored scenarios involving a hypothetical new variant $\mathrm{X}$ with the same basic reproduction number and generation time as the Delta variant, and at equal antigenic distance from Omicron and pre- 
Omicron variants. Accordingly, the relative risk of reinfection with variant $\mathrm{X}$ was assumed to be the same irrespective of whether an individual was primed with pre-Omicron or Omicron antigens. With an increased contact rate compared to Delta (Figure 3J), we found more opportunities for variant $\mathrm{X}$ to cause recuring epidemic waves in the explored ranges of parameters, primarily by escaping immunity conferred by pre-Omicron variants (Figure 3K). Emerging variants with such antigenic features need to be closely monitored in the future. On the other hand, if heterologous prime and boost infections (accounting for $45.7 \%$ of the population, Figure $3 \mathrm{~F}$ ) were found to elicit broadly protective antibodies and confer a high level of cross-protection against new variant X, only a small parameter space would be favorable to variant X epidemics (Figure $3 \mathrm{~K}$ ). Given that a large fraction of the world's population has been primed by vaccination or infection with preOmicron antigens, it is important to understand how heterologous boosting by Omicron may broaden the immune repertoire, and how this could translate into clinical protection against antigenically novel variants.

\section{Discussion}

15 For a period of 13 months, the PHIRST-C study carefully monitored SARS-CoV-2 infections in 222 households at a rural and an urban site in South Africa. These data provide a unique opportunity to characterize variant-specific shedding kinetics, transmission dynamics within the household, and the degree of immune protection conferred by prior infection before the Omicron surge. Longitudinal rRT-PCR data available for each infection episode at roughly 3-day resolution allowed for reconstruction of the intensity of SARS-CoV-2 exposures exerted on each household member based on $\mathrm{Ct}$ values. We found that individuals were more infectious in the RNA proliferation than clearance stage. Prior to the emergence of Omicron, substantial population immunity had accumulated through prior infection, with high and durable protection against symptomatic and asymptomatic reinfection, in line with prior findings $(30,31)$. These detailed cohort data allowed us to project the full scope of the Omicron epidemic and assess possible futures. Overall, even with a high degree of immunity post the Omicron wave (with over $90 \%$ of the 
population previously exposed to SARS-CoV-2 antigens), recurrence of past or antigenically novel variants is plausible, especially if post-Omicron behavioral changes increase contacts.

The disease burden of future SARS-CoV-2 epidemic waves depends on the intrinsic severity of the variants themselves as well as the level of protection conferred by pre-existing immunity (32). Current evidence suggests that while Omicron is able to evade immunity against infection to a significant degree, protection against severe outcomes remains high $(17,33,34)$. Our model's projections also suggest a low clinical burden of Omicron, with a greater than 10 -fold reduction in infection case ratio relative to prior waves (Figure 3E, Table S1). If existing immunity can sustain protection against severe outcomes over long timescales, the disease burden of future epidemic waves would be attenuated even if infections were widespread. However, if protection against severe outcomes waned over time, vaccine boosting would likely be needed to compensate for loss of protection.

As the PHIRST-C sampling scheme was not symptom-driven, it allowed us to capture shedding kinetics in both symptomatic and asymptomatic individuals. We observed that most infections $86.8 \%$ (581/669) were asymptomatic, but asymptomatically infected individuals transmitted the virus within their households. For context, in the South African winter seasons of 2017-2018, approximately half of the influenza infections within the PHIRST-C cohorts were asymptomatic (35). The transmission potential of asymptomatic or pre-symptomatic SARS-CoV-2 infections identified in our study is in sharp contrast with SARS-CoV-1, where most transmission occurs after symptom onset (36). It is also worth noting in our cohort data, prior infections, whether symptomatic or not, conferred durable protection against reinfection. We found that the shedding kinetics of SARS-CoV-2 were characterized by a rapid RNA proliferation stage until peak viral load, followed by a more gradual RNA clearance stage. The median duration of rRT-PCR positivity lasted 10.5 days (IQR 6.3 - 14.0 days) with median peak $\mathrm{Ct}=23.1$ (IQR 20.0 - 27.2), in agreement with other high-frequency sampling studies $(37,38)$. Reinfections had shorter durations of rRT-PCR positivity and lower shedding peaks compared to primary infections (Figure $2 \mathrm{H}-\mathrm{I}$ ), which would be expected to decrease the probability of onward transmission. Our findings align with reports of reduction in viral shedding among vaccine breakthroughs relative to primary infections, prior to the occurrence of the Omicron variant (39-42). Interestingly, we observed variation in infectiousness through the course of infection, after adjusting for $\mathrm{Ct}$ values, whereby 
an individual in the proliferation stage tends to be more infectious than one in the clearance stage. The post-peak decline in infectiousness coincides with the onset of adaptive immune responses that work to suppress the on-going infection (43). The observed decline in infectiousness in the RNA clearance stage also could be due to neutralization of some viral particles by antibodies, precluding productive transmission.

The peak of COVID-19 hospitalizations during the Omicron wave was lower than that during the Delta wave in South Africa (44), despite our model projecting a much higher infection rate for Omicron than Delta. This is compatible, however, with some lines of evidence suggesting a lower severity of the Omicron variant in naïve individuals, combined with robust infection and vaccineinduced immunity against severe Omicron disease $(17,45)$. However, it is important to stress that, in South Africa, immunity accumulated prior to the Omicron wave was mostly through prior infections, due to a delayed start and slow rollout of the vaccine campaign (46). As a result, the proportion of the population infected by pre-Omicron variants was substantially higher in South Africa than in countries that experienced faster vaccine rollout and/or effective mitigation 15 strategies.

A few caveats are worth noting. First, our findings about the persistence of infection-induced immunity are based on a 13-month study. The duration and quality of protective immunity over longer timescales remain open questions. Recent studies have found that antibody responses improve over time through affinity maturation $(47,48)$ and that long-lived plasma cells can be identified in the bone marrow at least one year after infection, suggesting that immunity conferred by infection or vaccination could be potent and durable against non-immune evasive variants (49). Persistent germinal center responses and durable $\mathrm{T}$ cell memory have also been observed among vaccine recipients (50-52). However, how the protection holds up against immune-evasive variant such as Omicron remains an outstanding question. Unfortunately, the PHIRST-C cohorts did not cover the Omicron wave, thus we could not directly measure immune protection at the individual level and relied on modelling of population-level dynamics. Post-Omicron serologic surveys following up the cohort population could provide deeper insight into the full impact of the Omicron wave. In our projections of SARS-CoV-2 resurgences, we did not consider waning explicitly since our cohort data did not support pronounced waning of infection-induced immunity. Accordingly, 
our projections are most relevant to short time scales, in the order of a few months. Interestingly, we find that resurgences are likely even over short time horizons.

A second caveat relates to missed infection episodes, despite frequent rRT-PCR testing. In total, 21\% (303407 person-days/1472400 person days) of the total person-days of observation were excluded from the regression during the entire study period due to missing nasal swab visits, missing serologic status, or experiencing an active infection episode. For example, 14\% (90/639) of individuals who seroconverted during the study lacked rRT-PCR confirmation of active infections (i.e., their first serologic test was negative, but they seroconverted later). This could possibly be due to 1) delayed seroconversion from infection episodes occurring prior to the first nasal specimen, 2) infection episodes that occurred during missed routine household visits, 3) a shorter duration of rRT-PCR positivity than the interval between consecutive nasal swabbing (3 days), 4) false positives in serology test results or 5) false negatives of the rRT-PCR assay specimen (due to specimen quality issues or detection limit of the rRT-PCR).

A third caveat relates to the simplicity of the contact structure in our transmission models. Projections of the trajectories of Delta, Omicron, and variant $\mathrm{X}$ did not address heterogeneity due to age-specific susceptibility, transmissibility, and contact patterns. Nor did we consider individual variation in infection and vaccine-derived protection. Heterogeneity in mixing patterns and immune protection could lead to a lower infection rate when compared to homogeneous models with the same basic reproduction number $(53,54)$, thus the size of the Omicron epidemic could be inflated in our projections. In addition, our size projections could be inflated if the Omicron's serial interval was shorter (corresponding to a lower basic reproduction number) than that the range of values explored. Population surveys on active infections during the Omicron wave as well as paired-sera surveys before and after the Omicron wave will be necessary to confirm the true scale of the Omicron epidemic.

25 In conclusion, our study provides an in-depth analysis of the kinetics of viral shedding, transmission dynamics, and persistence of immunity conferred by sequential exposures to different SARS-CoV-2 variants, and how these factors contribute to shaping the Omicron and post-Omicron phases. We found durable cross-protective immunity conferred by prior infection against preOmicron variants. However, Omicron successfully breached population immunity due to a 
combination immune escape and increased transmissibility, reinfecting a large fraction of the population and leaving a complex immune landscape in its aftermath. With increasing contacts as the Omicron wave subsides, several possible scenarios for SARS-CoV-2 recurrences are possible, involving both old and new variants. Further work on how immunity may strengthen and broaden upon sequential exposures with different variants and vaccination episodes will be important to clarify the next phase of the pandemic.

\section{References and Notes:}

1. L. F. Buss, C. A. Prete Jr, C. M. M. Abrahim, A. Mendrone Jr, T. Salomon, C. de Almeida-Neto, R. F. O. França, M. C. Belotti, M. P. S. S. Carvalho, A. G. Costa, M. A. E. Crispim, S. C. Ferreira, N. A. Fraiji, S. Gurzenda, C. Whittaker, L. T. Kamaura, P. L. Takecian, P. da Silva Peixoto, M. K. Oikawa, A. S. Nishiya, V. Rocha, N. A. Salles, A. A. de Souza Santos, M. A. da Silva, B. Custer, K. V. Parag, M. Barral-Netto, M. U. G. Kraemer, R. H. M. Pereira, O. G. Pybus, M. P. Busch, M. C. Castro, C. Dye, V. H. Nascimento, N. R. Faria, E. C. Sabino, Three-quarters attack rate of SARS$\mathrm{CoV}-2$ in the Brazilian Amazon during a largely unmitigated epidemic. Science. 371, 288-292 (2021).

2. T. R. Bhuiyan, J. Dent Hulse, S. T. Hegde, M. Akhtar, M. T. Islam, Z. Hasan Khan, I. I. Khan, S. Ahmed, M. M. Rashid, R. Rashid, E. S. Gurley, T. Shirin, A. I. Khan, A. S. Azman, F. Qadri, SARSCoV-2 seroprevalence in Chattogram, Bangladesh before the Delta surge, March-June 2021. bioRxiv (2021), , doi:10.1101/2021.07.16.21260611.

3. J. A. Huete-Pérez, K. C. Ernst, C. Cabezas-Robelo, L. Páiz-Medina, S. Silva, A. Huete, Prevalence and risk factors for SARS-CoV-2 infection in children with and without symptoms seeking care in Managua, Nicaragua: results of a cross-sectional survey. BMJ Open. 11, e051836 (2021).

4. C. Cohen, J. Kleynhans, A. von Gottberg, M. L. McMorrow, N. Wolter, J. N. Bhiman, J. Moyes, M. du Plessis, M. Carrim, A. Buys, N. A. Martinson, K. Kahn, S. Tollman, L. Lebina, F. Wafawanaka, J. du Toit, F. X. Gómez-Olivé, F. S. Dawood, T. Mkhencele, K. Sun, C. Viboud, S. Tempia, for the PHIRST group, SARS-CoV-2 incidence, transmission and reinfection in a rural and an urban setting: 
results of the PHIRST-C cohort study, South Africa, 2020-2021. bioRxiv (2021), , doi:10.1101/2021.07.20.21260855.

5. H. Tegally, E. Wilkinson, R. J. Lessells, J. Giandhari, S. Pillay, N. Msomi, K. Mlisana, J. N. Bhiman, A. von Gottberg, S. Walaza, V. Fonseca, M. Allam, A. Ismail, A. J. Glass, S. Engelbrecht, G. Van Zyl, W. Preiser, C. Williamson, F. Petruccione, A. Sigal, I. Gazy, D. Hardie, N.-Y. Hsiao, D. Martin, D. York, D. Goedhals, E. J. San, M. Giovanetti, J. Lourenço, L. C. J. Alcantara, T. de Oliveira, Sixteen novel lineages of SARS-CoV-2 in South Africa. Nat. Med. 27, 440-446 (2021).

6. H. Tegally, E. Wilkinson, M. Giovanetti, A. Iranzadeh, V. Fonseca, J. Giandhari, D. Doolabh, S. Pillay, E. J. San, N. Msomi, K. Mlisana, A. von Gottberg, S. Walaza, M. Allam, A. Ismail, T. Mohale, A. J. Glass, S. Engelbrecht, G. Van Zyl, W. Preiser, F. Petruccione, A. Sigal, D. Hardie, G. Marais, M. Hsiao, S. Korsman, M.-A. Davies, L. Tyers, I. Mudau, D. York, C. Maslo, D. Goedhals, S. Abrahams, O. Laguda-Akingba, A. Alisoltani-Dehkordi, A. Godzik, C. K. Wibmer, B. T. Sewell, J. Lourenço, L. C. J. Alcantara, S. L. Kosakovsky Pond, S. Weaver, D. Martin, R. J. Lessells, J. N. Bhiman, C. Williamson, T. de Oliveira, Emergence of a SARS-CoV-2 variant of concern with mutations in spike glycoprotein. Nature (2021), doi:10.1038/s41586-021-03402-9.

7. R. Viana, S. Moyo, D. G. Amoako, H. Tegally, C. Scheepers, C. L. Althaus, U. J. Anyaneji, P. A. Bester, M. F. Boni, M. Chand, W. T. Choga, R. Colquhoun, M. Davids, K. Deforche, D. Doolabh, L. du Plessis, S. Engelbrecht, J. Everatt, J. Giandhari, M. Giovanetti, D. Hardie, V. Hill, N.-Y. Hsiao, A. Iranzadeh, A. Ismail, C. Joseph, R. Joseph, L. Koopile, S. L. Kosakovsky Pond, M. U. G. Kraemer, L. Kuate-Lere, O. Laguda-Akingba, O. Lesetedi-Mafoko, R. J. Lessells, S. Lockman, A. G. Lucaci, A. Maharaj, B. Mahlangu, T. Maponga, K. Mahlakwane, Z. Makatini, G. Marais, D. Maruapula, K. Masupu, M. Matshaba, S. Mayaphi, N. Mbhele, M. B. Mbulawa, A. Mendes, K. Mlisana, A. Mnguni, T. Mohale, M. Moir, K. Moruisi, M. Mosepele, G. Motsatsi, M. S. Motswaledi, T. Mphoyakgosi, N. Msomi, P. N. Mwangi, Y. Naidoo, N. Ntuli, M. Nyaga, L. Olubayo, S. Pillay, B. Radibe, Y. Ramphal, U. Ramphal, J. E. San, L. Scott, R. Shapiro, L. Singh, P. Smith-Lawrence, W. Stevens, A. Strydom, K. Subramoney, N. Tebeila, D. Tshiabuila, J. Tsui, S. van Wyk, S. Weaver, C. K. Wibmer, E. Wilkinson, N. Wolter, A. E. Zarebski, B. Zuze, D. Goedhals, W. Preiser, F. Treurnicht, M. Venter, C. Williamson, O. G. Pybus, J. Bhiman, A. Glass, D. P. Martin, A. Rambaut, 
S. Gaseitsiwe, A. von Gottberg, T. de Oliveira, Rapid epidemic expansion of the SARS-CoV-2 Omicron variant in southern Africa. Nature (2022), doi:10.1038/d41586-021-03832-5.

8. C. A. B. Pearson, S. P. Silal, M. W. Z. Li, J. Dushoff, B. M. Bolker, S. Abbott, C. van Schalkwyk, N. G. Davies, R. C. Barnard, W. J. Edmunds, J. Bingham, G. Meyer-Rath, L. Jamieson, A. Glass, N. Wolter, N. Govender, W. S. Stevens, L. Scott, K. Mlisana, H. Moultrie, J. R. C. Pulliam, Bounding the levels of transmissibility \& immune evasion of the Omicron variant in South Africa. bioRxiv (2021), , doi:10.1101/2021.12.19.21268038.

9. Alaa Abdel Latif, Julia L. Mullen, Manar Alkuzweny, Ginger Tsueng, Marco Cano, Emily Haag, Jerry Zhou, Mark Zeller, Emory Hufbauer, Nate Matteson, Chunlei Wu, Kristian G. Andersen, Andrew I. Su, Karthik Gangavarapu, Laura D. Hughes, and the Center for Viral Systems Biology., Omicron Variant Report. outbreak.info (2022), (available at https://outbreak.info/situationreports/omicron?loc $=\mathrm{ZAF} \& \mathrm{loc}=\mathrm{GBR} \& \mathrm{loc}=\mathrm{USA} \&$ selected $=\mathrm{ZAF})$.

10. the Nextstrain team, Genomic epidemiology of novel coronavirus - Global subsampling. Nextstrain (2022), (available at https://nextstrain.org/ncov/gisaid/global).

11. J. R. C. Pulliam, C. van Schalkwyk, N. Govender, A. von Gottberg, C. Cohen, M. J. Groome, J. Dushoff, K. Mlisana, H. Moultrie, Increased risk of SARS-CoV-2 reinfection associated with emergence of the Omicron variant in South Africa. bioRxiv (2021), , doi:10.1101/2021.11.11.21266068.

12. Neil Ferguson, Azra Ghani, Anne Cori, Alexandra Hogan, Wes Hinsley, Erik Volz on behalf of the Imperial College COVID-19 response team, "Report 49 - Growth, population distribution and immune escape of Omicron in England" (Report 49, MRC Centre for Global Infectious Disease Analysis, Imperial College London, 2021), (available at https://www.imperial.ac.uk/mrc-globalinfectious-disease-analysis/covid-19/report-49-omicron/).

13. K. van der Straten, D. Guerra, M. J. van Gils, I. Bontjer, T. G. Caniels, H. D. G. van Willigen, E. Wynberg, M. Poniman, J. A. Burger, J. H. Bouhuijs, J. van Rijswijk, A. H. A. Lavell, B. Appelman, J. J. Sikkens, M. K. Bomers, A. X. Han, B. E. Nichols, M. Prins, H. Vennema, C. Reusken, M. D. de Jong, G. J. de Bree, C. A. Russell, D. Eggink, R. W. Sanders, Mapping the antigenic diversification of SARS-CoV-2. bioRxiv (2022), doi:10.1101/2022.01.03.21268582.

14. S. Cele, L. Jackson, D. S. Khoury, K. Khan, T. Moyo-Gwete, H. Tegally, J. E. San, D. Cromer, C. Scheepers, D. G. Amoako, F. Karim, M. Bernstein, G. Lustig, D. Archary, M. Smith, Y. Ganga, Z. 
Jule, K. Reedoy, S.-H. Hwa, J. Giandhari, J. M. Blackburn, B. I. Gosnell, S. S. Abdool Karim, W. Hanekom, NGS-SA, COMMIT-KZN Team, A. von Gottberg, J. N. Bhiman, R. J. Lessells, M.-Y. S. Moosa, M. P. Davenport, T. de Oliveira, P. L. Moore, A. Sigal, Omicron extensively but incompletely escapes Pfizer BNT162b2 neutralization. Nature (2021), doi:10.1038/s41586-021-

15. J. M. Carreño, H. Alshammary, J. Tcheou, G. Singh, A. Raskin, H. Kawabata, L. Sominsky, J. Clark, D. C. Adelsberg, D. Bielak, A. S. Gonzalez-Reiche, N. Dambrauskas, V. Vigdorovich, PSP/PARIS Study Group, K. Srivastava, D. N. Sather, E. M. Sordillo, G. Bajic, H. van Bakel, V. Simon, F. Krammer, Activity of convalescent and vaccine serum against SARS-CoV-2 Omicron. Nature (2021), doi:10.1038/s41586-022-04399-5.

16. E. Cameroni, J. E. Bowen, L. E. Rosen, C. Saliba, S. K. Zepeda, K. Culap, D. Pinto, L. A. VanBlargan, A. De Marco, J. di Iulio, F. Zatta, H. Kaiser, J. Noack, N. Farhat, N. Czudnochowski, C. Havenar-Daughton, K. R. Sprouse, J. R. Dillen, A. E. Powell, A. Chen, C. Maher, L. Yin, D. Sun, L. Soriaga, J. Bassi, C. Silacci-Fregni, C. Gustafsson, N. M. Franko, J. Logue, N. T. Iqbal, I. Mazzitelli, J. Geffner, R. Grifantini, H. Chu, A. Gori, A. Riva, O. Giannini, A. Ceschi, P. Ferrari, P. E. Cippà, A. Franzetti-Pellanda, C. Garzoni, P. J. Halfmann, Y. Kawaoka, C. Hebner, L. A. Purcell, L. Piccoli, M. S. Pizzuto, A. C. Walls, M. S. Diamond, A. Telenti, H. W. Virgin, A. Lanzavecchia, G. Snell, D. Veesler, D. Corti, Broadly neutralizing antibodies overcome SARS-CoV-2 Omicron antigenic shift. Nature (2021), doi:10.1038/s41586-021-04386-2.

17. UKHSA Genomics Cell UKHSA Outbreak Surveillance Team UKHSA Epidemiology Cell UKHSA Immunisations Team UKHSA Contact Tracing Data Team UKHSA Environmental Monitoring for Health Protection Team UKHSA SIREN Study Team UKHSA Public Health Incident Directors Contributions from the Variant Technical Group Members, "SARS-CoV-2 variants of concern and variants under investigation in England Technical briefing 34" (34, UK Health Security Agency, 2022), (available at https://assets.publishing.service.gov.uk/government/uploads/system/uploads/attachment_data/file/10 46853/technical-briefing-34-14-january-2022.pdf).

18. F. P. Lyngse, L. H. Mortensen, M. J. Denwood, L. E. Christiansen, C. H. Møller, R. L. Skov, K. Spiess, A. Fomsgaard, M. M. Lassaunière, M. Rasmussen, M. Stegger, C. Nielsen, R. N. Sieber, A. 
Omicron VOC transmission in Danish households. bioRxiv (2021), , doi:10.1101/2021.12.27.21268278.

19. A. C. Ulloa, S. A. Buchan, N. Daneman, K. A. Brown, Early estimates of SARS-CoV-2 Omicron variant severity based on a matched cohort study, Ontario, Canada. bioRxiv (2021), , doi:10.1101/2021.12.24.21268382.

20. WEEKLY EPIDEMIOLOGICAL BRIEF - NICD (2020), (available at https://www.nicd.ac.za/diseases-a-z-index/disease-index-covid-19/surveillance-reports/weeklyepidemiological-brief/).

21. J. A. Backer, D. Eggink, S. P. Andeweg, I. K. Veldhuijzen, N. van Maarseveen, K. Vermaas, B. Vlaemynck, R. Schepers, S. van den Hof, C. B. E. M. Reusken, J. Wallinga, Shorter serial intervals in SARS-CoV-2 cases with Omicron variant compared to Delta variant in the Netherlands, $13-19$ December 2021. bioRxiv (2022), , doi:10.1101/2022.01.18.22269217.

22. National Institute of Infectious Diseases Disease Control and Prevention Center, National Center for Global Health and Medicine, Active epidemiological investigation on SARS-CoV-2 infection caused by Omicron variant (Pango lineage B.1.1.529) in Japan: preliminary report on infectious period. 国 立感染症研究所 (2022), (available at https://www.niid.go.jp/niid/en/2019-ncov-e/10884-covid1966-en.html).

23. B. J. Willett, J. Grove, O. A. MacLean, C. Wilkie, N. Logan, G. D. Lorenzo, W. Furnon, S. Scott, M. Manali, A. Szemiel, S. Ashraf, E. Vink, W. Harvey, C. Davis, R. Orton, J. Hughes, P. Holland, V. Silva, D. Pascall, K. Puxty, A. da Silva Filipe, G. Yebra, S. Shaaban, M. T. G. Holden, R. M. Pinto, R. Gunson, K. Templeton, P. Murcia, A. H. Patel, J. Haughney, D. L. Robertson, M. Palmarini, S. Ray, E. C. Thomson, The COVID-19 DeplOyed VaccinE (DOVE) Cohort Study investigators, The COVID-19 Genomics UK (COG-UK) Consortium, The G2P-UK National Virology Consortium, The Evaluation of Variants Affecting Deployed COVID-19 Vaccines (EVADE) investigators, The hyper-transmissible SARS-CoV-2 Omicron variant exhibits significant antigenic change, vaccine escape and a switch in cell entry mechanism. bioRxiv (2022), , doi:10.1101/2022.01.03.21268111.

24. T. Braeye, L. Cornelissen, L. Catteau, F. Haarhuis, K. Proesmans, K. De Ridder, A. Djiena, R. Mahieu, F. De Leeuw, A. Dreuw, N. Hammami, S. Quoilin, H. Van Oyen, C. Wyndham-Thomas, D. 
Van Cauteren, Vaccine effectiveness against infection and onwards transmission of COVID-19: Analysis of Belgian contact tracing data, January-June 2021. Vaccine. 39, 5456-5460 (2021).

25. J. Kleynhans, S. Tempia, N. Wolter, A. von Gottberg, J. N. Bhiman, A. Buys, J. Moyes, M. L. McMorrow, K. Kahn, F. X. Gómez-Olivé, S. Tollman, N. A. Martinson, F. Wafawanaka, L. Lebina, J. du Toit, W. Jassat, M. Neti, M. Brauer, C. Cohen, PHIRST-C Group, SARS-CoV-2 Seroprevalence in a Rural and Urban Household Cohort during First and Second Waves of Infections, South Africa, July 2020-March 2021. Emerg. Infect. Dis. 27, 3020-3029 (2021).

26. A. Rössler, L. Riepler, D. Bante, D. von Laer, J. Kimpel, SARS-CoV-2 Omicron Variant Neutralization in Serum from Vaccinated and Convalescent Persons. N. Engl. J. Med. (2022), doi:10.1056/NEJMc2119236.

27. K. Khan, F. Karim, S. Cele, J. E. San, G. Lustig, H. Tegally, M. Bernstein, Y. Ganga, Z. Jule, K. Reedoy, N. Ngcobo, M. Mazibuko, N. Mthabela, Z. Mhlane, N. Mbatha, J. Giandhari, Y. Ramphal, T. Naidoo, N. Manickchund, N. Magula, S. S. Abdool Karim, G. Gray, W. Hanekom, A. von Gottberg, COMMIT-KZN Team, B. I. Gosnell, R. J. Lessells, P. L. Moore, T. de Oliveira, M.-Y. S. Moosa, A. Sigal, Omicron infection enhances neutralizing immunity against the Delta variant. medRxiv (2021), doi:10.1101/2021.12.27.21268439.

28. K. Spensley, S. Gleeson, P. Martin, T. Thomson, C. L. Clarke, G. Pickard, D. Thomas, S. P. McAdoo, P. Randell, P. Kelleher, R. Bedi, L. Lightstone, M. Prendecki, M. Willicombe, Comparison of vaccine effectiveness against the Omicron (B.1.1.529) variant in patients receiving haemodialysis. bioRxiv (2022), , doi:10.1101/2022.01.25.22269804.

29. H. Altarawneh, H. Chemaitelly, P. Tang, M. R. Hasan, S. Qassim, H. H. Ayoub, S. AlMukdad, H. M. Yassine, F. M. Benslimane, H. A. A. Khatib, P. Coyle, Z. A. Kanaani, E. A. Kuwari, A. Jeremijenko, A. H. Kaleeckal, A. N. Latif, R. M. Shaik, H. F. Abdul Rahim, G. K. Nasrallah, M. G. Al Kuwari, A. A. Butt, H. E. Al Romaihi, M. H. Al-Thani, A. A. Khal, R. Bertollini, L. J. AbuRaddad, Protection afforded by prior infection against SARS-CoV-2 reinfection with the Omicron variant. bioRxiv (2022), , doi:10.1101/2022.01.05.22268782.

30. V. J. Hall, S. Foulkes, F. Insalata, A. Saei, P. Kirwan, A. Atti, E. Wellington, J. Khawam, K. Munro, M. Cole, C. Tranquillini, A. Taylor-Kerr, N. Hettiarachchi, D. Calbraith, N. Sajedi, I. Milligan, Y. Themistocleous, D. Corrigan, L. Cromey, L. Price, S. Stewart, E. de Lacy, C. Norman, E. Linley, A. 
D. Otter, A. Semper, J. Hewson, The SIREN Study Group, M. A. Chand, C. S. Brown, T. Brooks, J. Islam, A. Charlett, S. Hopkins, medRxiv, in press.

31. C. H. Hansen, D. Michlmayr, S. M. Gubbels, K. Mølbak, S. Ethelberg, Assessment of protection against reinfection with SARS-CoV-2 among 4 million PCR-tested individuals in Denmark in 2020: a population-level observational study. Lancet (2021), doi:10.1016/S0140-6736(21)00575-4.

32. A. Sigal, Milder disease with Omicron: is it the virus or the pre-existing immunity? Nat. Rev. Immunol. (2022), doi:10.1038/s41577-022-00678-4.

33. M. G. Thompson, Effectiveness of a Third Dose of mRNA Vaccines Against COVID-19-Associated Emergency Department and Urgent Care Encounters and Hospitalizations Among Adults During Periods of Delta and Omicron Variant Predominance — VISION Network, 10 States, August 2021January 2022. MMWR Morb. Mortal. Wkly. Rep. 71 (2022), doi:10.15585/mmwr.mm7104e3.

34. J. A. Lewnard, V. X. Hong, M. M. Patel, R. Kahn, M. Lipsitch, S. Y. Tartof, Clinical outcomes among patients infected with Omicron (B.1.1.529) SARS-CoV-2 variant in southern California. bioRxiv (2022), , doi:10.1101/2022.01.11.22269045.

35. C. Cohen, J. Kleynhans, J. Moyes, M. L. McMorrow, F. K. Treurnicht, O. Hellferscee, A. Mathunjwa, A. von Gottberg, N. Wolter, N. A. Martinson, K. Kahn, L. Lebina, K. Mothlaoleng, F. Wafawanaka, F. X. Gómez-Olivé, T. Mkhencele, A. Mathee, S. Piketh, B. Language, S. Tempia, PHIRST group, Asymptomatic transmission and high community burden of seasonal influenza in an urban and a rural community in South Africa, 2017-18 (PHIRST): a population cohort study. Lancet Glob Health. 9, e863-e874 (2021).

36. C. Fraser, S. Riley, R. M. Anderson, N. M. Ferguson, Factors that make an infectious disease outbreak controllable. Proc. Natl. Acad. Sci. U. S. A. 101, 6146-6151 (2004).

37. S. M. Kissler, J. R. Fauver, C. Mack, S. W. Olesen, C. Tai, K. Y. Shiue, C. C. Kalinich, S. Jednak, I. M. Ott, C. B. F. Vogels, J. Wohlgemuth, J. Weisberger, J. DiFiori, D. J. Anderson, J. Mancell, D. D. Ho, N. D. Grubaugh, Y. H. Grad, SARS-CoV-2 viral dynamics in acute infections. bioRxiv (2020), doi:10.1101/2020.10.21.20217042.

38. T. C. Jones, G. Biele, B. Mühlemann, T. Veith, J. Schneider, J. Beheim-Schwarzbach, T. Bleicker, J. Tesch, M. L. Schmidt, L. E. Sander, F. Kurth, P. Menzel, R. Schwarzer, M. Zuchowski, J. Hofmann, 
A. Krumbholz, A. Stein, A. Edelmann, V. M. Corman, C. Drosten, Estimating infectiousness throughout SARS-CoV-2 infection course. Science (2021), doi:10.1126/science.abi5273.

39. S. D. Pollett, S. A. Richard, A. C. Fries, M. P. Simons, K. Mende, T. Lalani, T. Lee, S. Chi, R. Mody, C. Madar, A. Ganesan, D. T. Larson, C. J. Colombo, R. Colombo, E. C. Samuels, C. C. Broder, E. D. Laing, D. R. Smith, D. Tribble, B. K. Agan, T. H. Burgess, The SARS-CoV-2 mRNA vaccine breakthrough infection phenotype includes significant symptoms, live virus shedding, and viral genetic diversity. Clin. Infect. Dis. (2021), doi:10.1093/cid/ciab543.

40. M. G. Thompson, J. L. Burgess, A. L. Naleway, H. Tyner, S. K. Yoon, J. Meece, L. E. W. Olsho, A. J. Caban-Martinez, A. L. Fowlkes, K. Lutrick, H. C. Groom, K. Dunnigan, M. J. Odean, K. Hegmann, E. Stefanski, L. J. Edwards, N. Schaefer-Solle, L. Grant, K. Ellingson, J. L. Kuntz, T. Zunie, M. S. Thiese, L. Ivacic, M. G. Wesley, J. Mayo Lamberte, X. Sun, M. E. Smith, A. L. Phillips, K. D. Groover, Y. M. Yoo, J. Gerald, R. T. Brown, M. K. Herring, G. Joseph, S. Beitel, T. C. Morrill, J. Mak, P. Rivers, B. P. Poe, B. Lynch, Y. Zhou, J. Zhang, A. Kelleher, Y. Li, M. Dickerson, E. Hanson, K. Guenther, S. Tong, A. Bateman, E. Reisdorf, J. Barnes, E. AzzizBaumgartner, D. R. Hunt, M. L. Arvay, P. Kutty, A. M. Fry, M. Gaglani, Prevention and Attenuation of Covid-19 with the BNT162b2 and mRNA-1273 Vaccines. N. Engl. J. Med. (2021), doi:10.1056/NEJMoa2107058.

41. M. Levine-Tiefenbrun, I. Yelin, R. Katz, E. Herzel, Z. Golan, L. Schreiber, T. Wolf, V. Nadler, A. Ben-Tov, J. Kuint, S. Gazit, T. Patalon, G. Chodick, R. Kishony, Initial report of decreased SARSCoV-2 viral load after inoculation with the BNT162b2 vaccine. Nat. Med. 27, 790-792 (2021).

42. G. Regev-Yochay, S. Amit, M. Bergwerk, M. Lipsitch, E. Leshem, R. Kahn, Y. Lustig, C. Cohen, R. Doolman, A. Ziv, I. Novikov, C. Rubin, I. Gimpelevich, A. Huppert, G. Rahav, A. Afek, Y. Kreiss, Decreased infectivity following BNT162b2 vaccination: A prospective cohort study in Israel. The Lancet Regional Health - Europe. 7, 100150 (2021).

43. L. Guo, L. Ren, S. Yang, M. Xiao, D. Chang, F. Yang, C. S. Dela Cruz, Y. Wang, C. Wu, Y. Xiao, L. Zhang, L. Han, S. Dang, Y. Xu, Q.-W. Yang, S.-Y. Xu, H.-D. Zhu, Y.-C. Xu, Q. Jin, L. Sharma, L. Wang, J. Wang, Profiling Early Humoral Response to Diagnose Novel Coronavirus Disease (COVID-19). Clin. Infect. Dis. 71, 778-785 (2020).

44. The National Institute For Communicable Diseases Of South Africa, "NICD National COVID-19 Hospital Surveillance” (The National Institute For Communicable Diseases Of South Africa, 2022), 
(available at https://www.nicd.ac.za/wp-content/uploads/2022/02/NICD-COVID-19-Daily-SentinelHospital-Surveillance-report-National-20220203.pdf).

45. N. Wolter, W. Jassat, S. Walaza, R. Welch, H. Moultrie, M. Groome, D. G. Amoako, J. Everatt, J. N. Bhiman, C. Scheepers, N. Tebeila, N. Chiwandire, M. du Plessis, N. Govender, A. Ismail, A. Glass, K. Mlisana, W. Stevens, F. K. Treurnicht, Z. Makatini, N.-Y. Hsiao, R. Parboosing, J. Wadula, H. Hussey, M.-A. Davies, A. Boulle, A. von Gottberg, C. Cohen, Early assessment of the clinical severity of the SARS-CoV-2 omicron variant in South Africa: a data linkage study. Lancet (2022), doi:10.1016/S0140-6736(22)00017-4.

46. South Africa National Department Of Health, Latest Vaccine Statistics (South Africa). SA Corona Virus Online Portal (2021), (available at https://sacoronavirus.co.za/latest-vaccine-statistics/).

47. A. Cho, F. Muecksch, D. Schaefer-Babajew, Z. Wang, S. Finkin, C. Gaebler, V. Ramos, M. Cipolla, P. Mendoza, M. Agudelo, E. Bednarski, J. DaSilva, I. Shimeliovich, J. Dizon, M. Daga, K. G. Millard, M. Turroja, F. Schmidt, F. Zhang, T. B. Tanfous, M. Jankovic, T. Y. Oliveria, A. Gazumyan, M. Caskey, P. D. Bieniasz, T. Hatziioannou, M. C. Nussenzweig, Anti-SARS-CoV-2 receptor-binding domain antibody evolution after mRNA vaccination. Nature. 600, 517-522 (2021).

48. F. Muecksch, Y. Weisblum, C. O. Barnes, F. Schmidt, D. Schaefer-Babajew, Z. Wang, J. C. C Lorenzi, A. I. Flyak, A. T. DeLaitsch, K. E. Huey-Tubman, S. Hou, C. A. Schiffer, C. Gaebler, J. Da Silva, D. Poston, S. Finkin, A. Cho, M. Cipolla, T. Y. Oliveira, K. G. Millard, V. Ramos, A. Gazumyan, M. Rutkowska, M. Caskey, M. C. Nussenzweig, P. J. Bjorkman, T. Hatziioannou, P. D. Bieniasz, Affinity maturation of SARS-CoV-2 neutralizing antibodies confers potency, breadth, and resilience to viral escape mutations. Immunity. 54, 1853-1868.e7 (2021).

49. J. S. Turner, W. Kim, E. Kalaidina, C. W. Goss, A. M. Rauseo, A. J. Schmitz, L. Hansen, A. Haile, M. K. Klebert, I. Pusic, J. A. O’Halloran, R. M. Presti, A. H. Ellebedy, SARS-CoV-2 infection induces long-lived bone marrow plasma cells in humans. Nature. 595, 421-425 (2021).

50. J. S. Turner, J. A. O’Halloran, E. Kalaidina, W. Kim, A. J. Schmitz, J. Q. Zhou, T. Lei, M. Thapa, R. E. Chen, J. B. Case, F. Amanat, A. M. Rauseo, A. Haile, X. Xie, M. K. Klebert, T. Suessen, W. D. Middleton, P.-Y. Shi, F. Krammer, S. A. Teefey, M. S. Diamond, R. M. Presti, A. H. Ellebedy, 
SARS-CoV-2 mRNA vaccines induce persistent human germinal centre responses. Nature (2021), doi:10.1038/s41586-021-03738-2.

51. J. Mateus, J. M. Dan, Z. Zhang, C. R. Moderbacher, M. Lammers, B. Goodwin, A. Sette, S. Crotty, D. Weiskopf, Low dose mRNA-1273 COVID-19 vaccine generates durable T cell memory and antibodies enhanced by pre-existing crossreactive T cell memory. bioRxiv (2021), , doi:10.1101/2021.06.30.21259787.

52. J. M. Dan, J. Mateus, Y. Kato, K. M. Hastie, E. D. Yu, C. E. Faliti, A. Grifoni, S. I. Ramirez, S. Haupt, A. Frazier, C. Nakao, V. Rayaprolu, S. A. Rawlings, B. Peters, F. Krammer, V. Simon, E. O. Saphire, D. M. Smith, D. Weiskopf, A. Sette, S. Crotty, Immunological memory to SARS-CoV-2 assessed for up to 8 months after infection. Science. 371 (2021), doi:10.1126/science.abf4063.

53. D. Mistry, M. Litvinova, A. Pastore Y Piontti, M. Chinazzi, L. Fumanelli, M. F. C. Gomes, S. A. Haque, Q.-H. Liu, K. Mu, X. Xiong, M. E. Halloran, I. M. Longini Jr, S. Merler, M. Ajelli, A. Vespignani, Inferring high-resolution human mixing patterns for disease modeling. Nat. Commun. $12,323(2021)$

54. T. Britton, F. Ball, P. Trapman, A mathematical model reveals the influence of population heterogeneity on herd immunity to SARS-CoV-2. Science. 369, 846-849 (2020).

55. C. Cohen, M. L. McMorrow, N. A. Martinson, K. Kahn, F. K. Treurnicht, J. Moyes, T. Mkhencele, O. Hellferscee, L. Lebina, M. Moroe, K. Motlhaoleng, F. X. Gómez-Olivé, R. Wagner, S. Tollman, F. Wafawanaka, S. Ngobeni, J. Kleynhans, A. Mathunjwa, A. Buys, L. Maake, N. Wolter, M. Carrim, S. Piketh, B. Language, A. Mathee, A. von Gottberg, S. Tempia, for the PHIRST group, Cohort Profile: a Prospective Household cohort study of Influenza, Respiratory Syncytial virus, and other respiratory pathogens community burden and Transmission dynamics in South Africa (PHIRST), 2016-2018. bioRxiv (2021), , doi:10.1101/2021.01.06.21249313.

56. Elecsys ${ }^{\circledR}$ Anti-SARS-CoV-2, (available at https://diagnostics.roche.com/us/en/products/params/elecsys-anti-sars-cov-2.html).

57. M. J. Peluso, S. Takahashi, J. Hakim, J. D. Kelly, L. Torres, N. S. Iyer, K. Turcios, O. Janson, S. E. Munter, C. Thanh, C. C. Nixon, R. Hoh, V. Tai, E. A. Fehrman, Y. Hernandez, M. A. Spinelli, M. Gandhi, M.-A. Palafox, A. Vallari, M. A. Rodgers, J. Prostko, J. Hackett, L. Trinh, T. Wrin, C. J. Petroplolous, C. Y. Chiu, P. J. Norris, C. DiGermanio, M. Stone, M. P. Busch, S. K. Elledge, X. X. Zhou, J. A. Wells, A. Shu, T. W. Kurtz, J. E. Pak, W. Wu, P. D. Burbelo, J. I. Cohen, R. L. 
Rutishauser, J. N. Martin, S. G. Deeks, T. J. Henrich, I. Rodriguez-Barraquer, B. Greenhouse, medRxiv, in press.

58. CDC, Frequently Asked Questions about Coronavirus (COVID-19) for Laboratories (2021), (available at https://www.cdc.gov/coronavirus/2019-ncov/lab/faqs.html).

59. Public Health England, Cycle threshold (Ct) in SARS-CoV-2 RT-PCR (2020), (available at https://www.gov.uk/government/publications/cycle-threshold-ct-in-sars-cov-2-rt-pcr).

60. S. M. Kissler, J. R. Fauver, C. Mack, C. G. Tai, M. I. Breban, A. E. Watkins, R. M. Samant, D. J. Anderson, D. D. Ho, N. D. Grubaugh, Y. H. Grad, Densely sampled viral trajectories suggest longer duration of acute infection with B.1.1.7 variant relative to non-B.1.1.7 SARS-CoV-2. bioRxiv (2021), , doi:10.1101/2021.02.16.21251535.

61. M. Kang, H. Xin, J. Yuan, S. T. Ali, Z. Liang, J. Zhang, T. Hu, E. H. Y. Lau, Y. Zhang, M. Zhang, B. J. Cowling, Y. Li, P. Wu, Transmission dynamics and epidemiological characteristics of Delta variant infections in China. bioRxiv (2021), , doi:10.1101/2021.08.12.21261991.

62. A. Marc, M. Kerioui, F. Blanquart, J. Bertrand, O. Mitjà, M. Corbacho-Monné, M. Marks, J. Guedj, Quantifying the relationship between SARS-CoV-2 viral load and infectiousness. Elife. 10 (2021), doi:10.7554/eLife.69302.

63. Douglas Bates and Martin Machler and Ben Bolker and Steve Walker, Fitting Linear Mixed-Effects Models Using lme4. Journal of Statistical Software. 67, 1-48 (2015).

64. R. A. Neher, R. Dyrdak, V. Druelle, E. B. Hodcroft, J. Albert, Potential impact of seasonal forcing on a SARS-CoV-2 pandemic. Swiss Med. Wkly. 150, w20224 (2020).

Acknowledgment: The findings and conclusions in this report are those of the authors and do not necessarily represent the official position of the NIH or the U.S. Centers for Disease Control and Prevention. Funding: This work was supported by the National Institute for Communicable Diseases of the National Health Laboratory Service and the U.S. Centers for Disease Control and Prevention [cooperative agreement number: 6 U01IP001048-04-02] and Wellcome Trust (grant number 221003/Z/20/Z) in collaboration with the Foreign, Commonwealth and Development Office, United Kingdom. Author contributions: KS, ST, JK, AvG, MLM, NW, JM, NAM, KK, 
STo, LL, CV, CC designed the experiments. CC, JK, and ST accessed and verified the underlying data. ST, JK, AvG, MLM, NW, JNB, JM, MdP, MC, AB, NAM, KK, STo, LL, FW, JdT, FXG, FSD, TMK, CC collected the data and performed laboratory experiments. KS, ST, JK, AvG, MLM, NW, JNB, JM, MdP, MC, AB, NAM, KK, STo, LL, FW, JdT, FXG, FSD, TMK, CV, and CC analyzed the data and interpreted the results. KS, ST, JK, AvG, CV, and CC drafted the manuscript. All authors critically reviewed the Article. All authors had access to all the data reported in the study. Competing interests: $\mathrm{CC}$ has received grant support from Sanofi Pasteur, Advanced Vaccine Initiative, and payment of travel costs from Parexel. AvG has received grant support from Sanofi Pasteur, Pfizer related to pneumococcal vaccine, CDC and the Bill \& Melinda Gates Foundation. NW reports grants from Sanofi Pasteur and the Bill \& Melinda Gates Foundation. NAM has received a grant to his institution from Pfizer to conduct research in patients with pneumonia and from Roche to collect specimens to assess a novel TB assay. JM has received grant support from Sanofi Pasteur. Ethics statement: The PHIRST-C protocol was approved by the University of Witwatersrand Human Research Ethics Committee (Reference 150808) and the U.S. Centers for Disease Control and Prevention's Institutional Review Board relied on the local review (\#6840). The protocol was registered on clinicaltrials.gov on 6 August 2015 and updated on 30 December 2020 (https://clinicaltrials.gov/ct2/show/NCT02519803). Participants receive grocery store vouchers of ZAR50 (USD 3) per visit to compensate for time required for specimen collection and interview. 

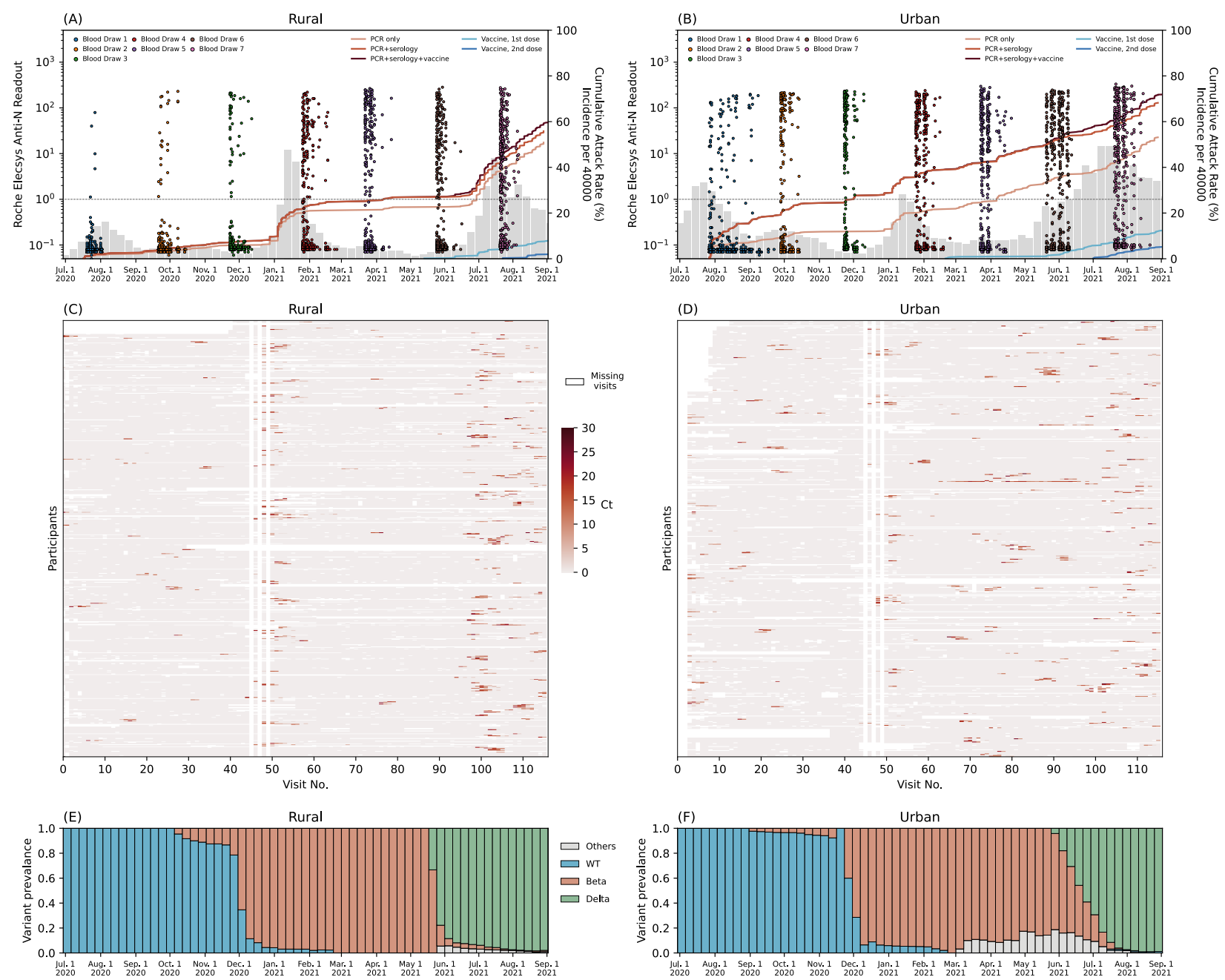

Fig. 1. PHIRST-C study June 2020 - September 2021, description of the epidemiology of SARS-CoV-2 in the two study sites, along with serology and rRT-PCR data. (A) Dots in different colors represent the timing and the readouts (axis on the left) of Roche Elecsys Anti-SARS-CoV-2 assay of serum specimens collected from 4 different blood draws of the rural cohort. The dash line is the positive cutoff of the Roche Elecsys Anti-SARS-CoV-2 assay, above which a specimen is considered sero-positive. The red lines (from light to dark) are the cumulative SARS-CoV-2 variant exposures (axis on the right) over time, captured by positive rRT-PCR of mid-turbinate nasal swab samples only; by either positive serum antibody or positive mid-turbinate nasal swabs by rRT-PCR, and by either positive serum antibody or positive mid-turbinate nasal swabs by rRT-PCR or at least one dose of vaccine. The light and dark blue lines are the cumulative fraction of population receiving a $1^{\text {st }}$ and $2^{\text {nd }}$ dose of vaccine. The grey bars are the weekly SARS-CoV-2 incidence per 40,000 population (sharing the same axis on the right) captured by the surveillance system of Ehlanzeni District in Mpumalanga Province, where the rural site is located. (B) Same as (A) but for the urban site of Klerksdorp in the Dr Kenneth Kaunda District, North West Province. (C) rRT-PCR test results for all mid-turbinate nasal specimens collected from individuals in the rural cohort over 80 visits during the 13-month study period. Color white indicates missing specimens; color red indicates the Ct value of the rRT-PCR test, the darker the red color, the lower the Ct value. (D) Same as (C) but for the urban cohort. (E) The bi-monthly relative prevalence of D614G mutation, Beta, Delta and other variants over time at the rural site. (F) Same as (E) but for the urban site. 


\begin{tabular}{|c|c|c|}
\hline & Rural & Urban \\
\hline Characteristics & No. $(\%)$ & No. $(\%)$ \\
\hline All & $643(100)$ & $557(100)$ \\
\hline \multicolumn{3}{|l|}{ Age group, in years } \\
\hline $0-5$ & $99(15)$ & $55(10)$ \\
\hline $6-18$ & $299(47)$ & $211(38)$ \\
\hline $19-65$ & $218(34)$ & $260(47)$ \\
\hline$>65$ & $27(4)$ & $31(5)$ \\
\hline \multicolumn{3}{|l|}{ Sex } \\
\hline Male & $234(36)$ & $249(45)$ \\
\hline Female & $409(64)$ & $308(55)$ \\
\hline \multicolumn{3}{|l|}{ Household size } \\
\hline$<4$ & $44(7)$ & $62(11)$ \\
\hline $4-6$ & $282(44)$ & $296(53)$ \\
\hline $7-10$ & $257(40)$ & $141(25)$ \\
\hline$>10$ & $60(9)$ & $58(10)$ \\
\hline \multicolumn{3}{|l|}{ BMI } \\
\hline Underweight & $259(40)$ & $140(25)$ \\
\hline Healthy weight & $206(32)$ & $192(35)$ \\
\hline Overweight & $93(14)$ & $103(18)$ \\
\hline Obesity & $85(13)$ & $120(22)$ \\
\hline Unknown & $0(0)$ & $2(0)$ \\
\hline \multicolumn{3}{|l|}{ HIV status } \\
\hline Negative & $520(81)$ & $451(81)$ \\
\hline PLWH*: CD4 < 200 & $5(1)$ & $9(2)$ \\
\hline $\mathrm{PLWH}^{*}: \mathrm{CD} 4 \geqslant 200$ & $75(12)$ & $78(14)$ \\
\hline Unknown & $43(7)$ & $19(3)$ \\
\hline \multicolumn{3}{|l|}{ Vaccination status } \\
\hline None & $593(92)$ & $488(88)$ \\
\hline $\mathrm{J} \& \mathrm{~J}$ & $12(2)$ & $9(2)$ \\
\hline Pfizer first dose only & $27(4)$ & $32(6)$ \\
\hline Pfizer first and second doses & $11(2)$ & $28(5)$ \\
\hline
\end{tabular}

Table 1: PHIRST-C study June 2020 - August 2021, characteristics of the population and SARS-CoV-2 infections at two study sites, South Africa. *PLWH: persons living with HIV 

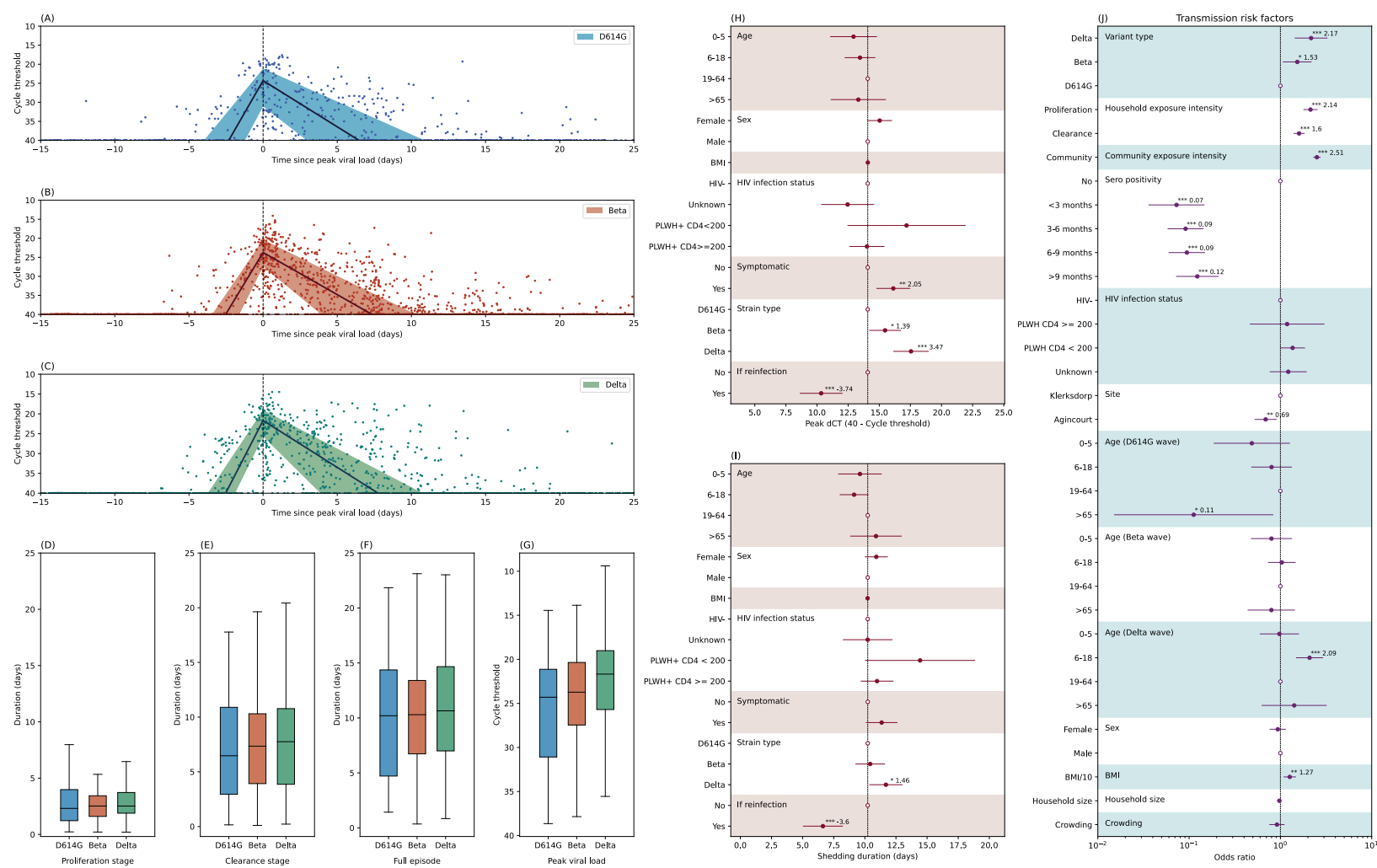

Fig. 2. (A) Characterization of the RNA shedding kinetics for D614G infections. The blue dots are longitudinal $\mathrm{Ct}$ values observation for each infection episode, aligned based on the estimated timing of peak $d C t$ (difference in $\mathrm{Ct}$ values between the result of a particular specimen and the detection limit of the rRT-PCR). The blue line is the population median of all individual fits, and the blue shade is the interquartile range. (B) Same as (A) but for Beta variant. (C) Same as (A) but for Delta variant. Distribution of the estimated duration of the viral RNA proliferation stage. (D) Distribution of the estimated duration of the viral RNA proliferation stage for D614G, Beta, and Delta variants. Boxplots show median, interquartile range, minimum and maximum of the distribution (E) Same as (D) but for the distribution of the estimated duration of the viral RNA clearance stage. (F) Same as (D) but for the distribution of the estimated full duration (proliferation stage + clearance stage) of rRT-PCR positivity. (G) Same as (D) but for the distribution of the estimated peak $d C t$. $(\mathrm{H})$ The association between peak $d C t$ and age, sex BMI, HIV infection status, symptom presentation, variant type, and prior infection history, based on gaussian multiple regression. Regression coefficients along with $95 \%$ CIs are reported as solid dots and horizontal lines relative to the value of the regression intercept. The hollow dots are reference class for each of the categorical variable. (I) Same as (H) but for shedding duration. (J) Logistic regression analyses on risk factors associated with infection acquisition. Odds ratios (OR) along with $95 \%$ CIs are reported as solid dots and horizontal lines. The hollow dots are reference class for each of the categorical variable. Protection is measured as $1-O R$.

*Indicates $\mathrm{p}<0.05$; ** indicates $\mathrm{p}<0.01$; *** indicates $\mathrm{p}<0.001$. Abbreviations: HIV- (HIV-uninfected individuals), PLWH+ CD4 $<200$ (Persons living with HIV, CD4+ T cell count under 200 cells/ml), PLWH+ CD4 >=200 (Persons living with HIV, CD4+ T cell count equal or above 200 cells $/ \mathrm{ml}$ ). 

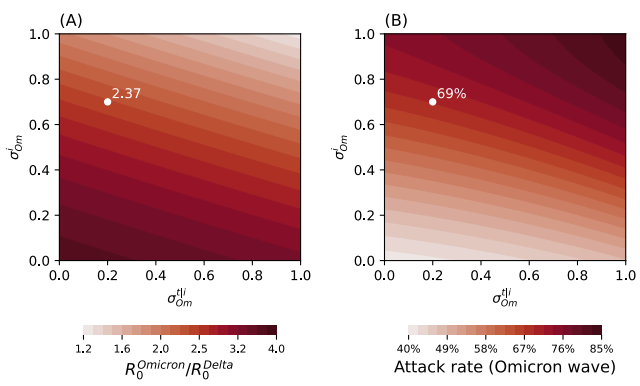

(F)

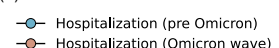

$-0-$ Hospitalization (pre Omicron)
$\rightarrow-$ Hospitalization (Omicron wave)

Cases (pre Omicron)
$\square$ Infections (pre Omicron)
Infections (Omicron wave)
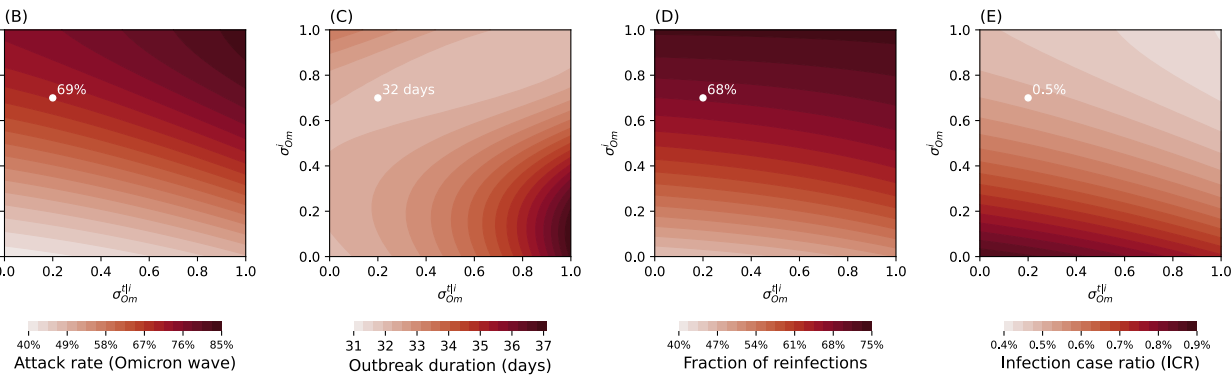

$40 \% \quad 47 \% \quad 54 \% \quad 61 \% \quad 68 \% \quad 75 \%$
Fraction of reinfections $0.4 \% \quad 0.5 \% \quad 0.6 \% \quad 0.7 \% \quad 0.8 \% \quad 0.9 \%$
Infection case ratio (ICR)
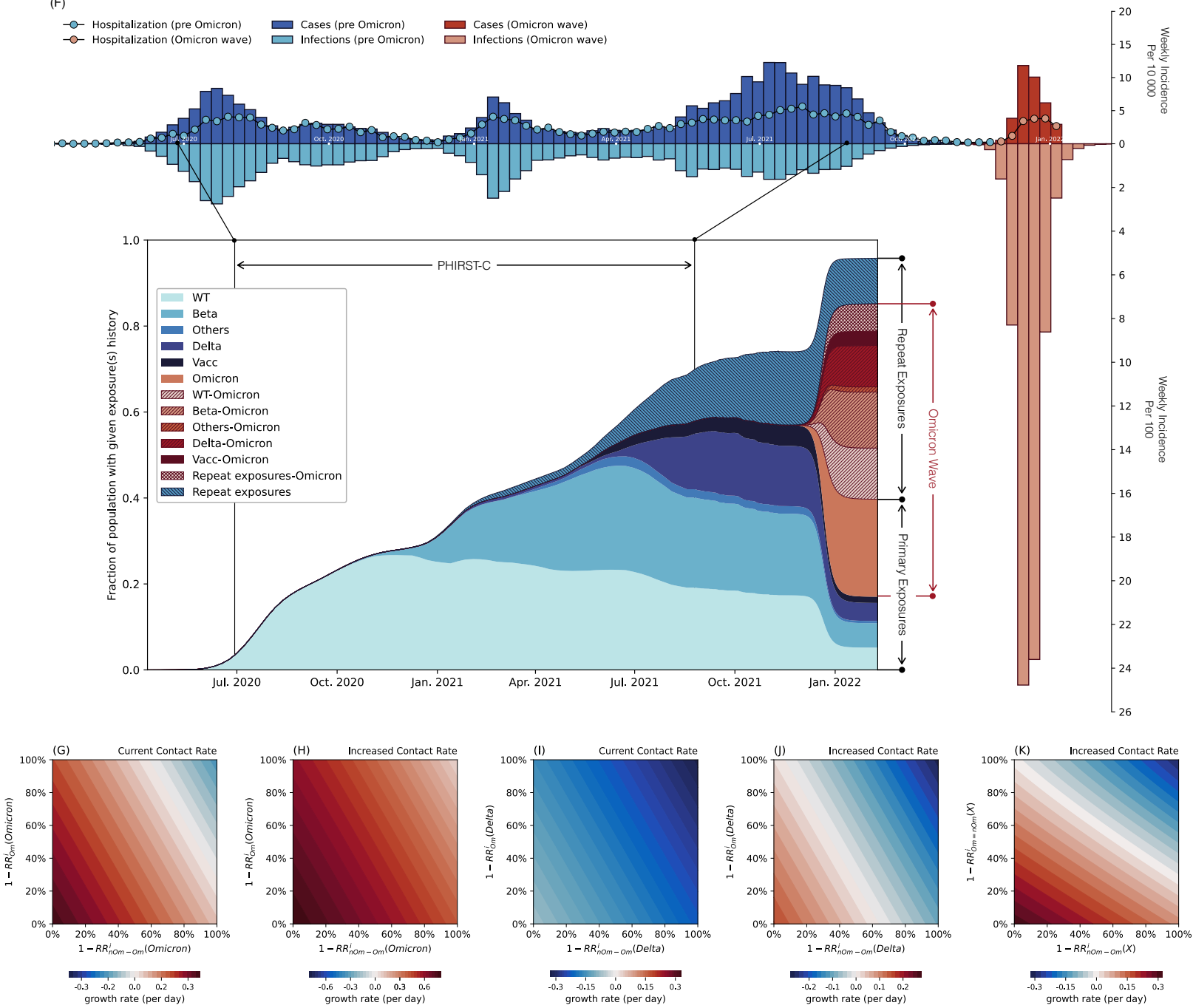

Fig. 3. (A) Phase diagram of estimated reproduction number ratio between Omicron and Delta $\left(R_{0}^{\text {Omicron }} / R_{0}^{\text {Delta }}\right)$ as a function of immune escape parameters $\left(\sigma_{O m}^{i}=\right.$ escape on infection) and $\sigma_{O m}^{t \mid i}$ (escape on transmission reduction conditional on infection). Parameters shown are those that matched the observed growth advantage of Omicron over Delta and the timing of the Omicron peak in the urban district of the PHIRST-C study (B) Phase diagram of the infection rate of the Omicron wave as a function of $\sigma_{O m}^{i}$ and $\sigma_{O m}^{t \mid i}$ and the corresponding $R_{0}^{\text {Omicron }} / R_{0}^{\text {Delta }}$ in (A). (C) Phase diagram of the epidemic duration of the Omicron wave as a function of $\sigma_{O m}^{i}$ and $\sigma_{O m}^{t \mid i}$ and the corresponding $R_{0}^{\text {Omicron }} / R_{0}^{\text {Delta }}$ in (A). (D) Phase diagram of the fraction of reinfections of the Omicron wave as a 
function of $\sigma_{O m}^{i}$ and $\sigma_{O m}^{t \mid i}$ and the corresponding $R_{0}^{\text {Omicron }} / R_{0}^{\text {Delta }}$ in (A). (E) For a reference scenario of immune escape where $\sigma_{O m}^{i}=0.7$ and $\sigma_{O m}^{t \mid i}=0.2$ (white dot in Figure 3A), we plot the phase diagram of realized ICR ratio during the Omicron wave vs the D614G wave (first wave) as a function of the ICR ratio of the intrinsic (primary infections only) Omicron ICR vs that of D614G and $R R^{c \mid i}$ (reduction in risk of hospitalization given prior infections) (F) Reconstruction of infection time series and exposure histories by variant: Top panel y axis upwards: Weekly incidence per 10,000 individuals of SARS-CoV-2 cases reported to the District till January 2022, in the period before Omicron (dark blue bars) and during Omicron (dark red bars). Top panel y axis downwards: Weekly incidence per 100 individuals of SARS-CoV-2 infections reconstructed based on PHRIST-C data (prior to September 2021) and estimated using Delta/Omicron-specific transmission models from September 2021 to the end of the Omicron wave at the end of January 2022. Pre-Omicron infections are in light blue and Omicron infections are in light red. For the top panel, the y axis upwards and downwards have different scales (by a factor of 100). Insert panel: the prevalence of the population with specific SARS-CoV-2 antigen exposure histories. (G-K): Possible post-Omicron futures, exploring potential resurgences of Omicron, Delta, or a new variant $\mathrm{X}$, under different contact scenarios. Projections are based on the reconstructed immune histories at the end of January 2022 shown in Fig 3F (i.e., given a reference scenario for Omicron's immune escape $\sigma_{O m}^{i}=0.7$ and $\sigma_{O m}^{t \mid i}=0.2$ ). (G) Risk of recurrence of Omicron: phase diagram of the growth rate of Omicron in a recurrent wave, as a function of the level of protection conferred by Omicron primary infections against Omicron $1-R R_{O m}^{i}(\mathrm{Om})$, and the level of protection conferred by Omicron reinfections/breakthroughs against Omicron $1-R R_{n O m-O m}^{i}(\mathrm{Om})$. Contact rates are assumed to remain the same as during the Omicron wave. $(\mathrm{H})$ same as $(\mathrm{G})$ but assuming the contact rate is twice of that during the Omicron wave. (I) Risk of recurrence of Delta: Phase diagram of the growth rate of Delta, after the initial Omicron wave has subsided, as a function of the level of protection conferred by Omicron primary infection against Delta $1-R R_{O m}^{i}($ Delta $)$, and the level of protection conferred by Omicron reinfections/breakthroughs against Delta $1-R R_{\text {nOm-Om }}^{i}($ Delta). Contact rates are assumed to remain the same as during the Omicron wave. (J) same as (I) but assuming the contact rate is twice of that during the Omicron wave. (K) Risk of occurrence of hypothetical new variant $\mathrm{X}$, where $\mathrm{X}$ is at equal antigenic distance of Delta and Omicron: Phase diagram of the growth rate of variant $\mathrm{X}$, after the initial Omicron wave has subsided, as a function of the level of protection conferred by any variant primary infection on infection with $\mathrm{X}\left(1-R R_{O m=n O m}^{i}(X)\right.$, assuming Omicron and pre-Omicron infections confer the same level of protection against X), and the level of protection conferred by Omicron reinfections/breakthroughs on $\mathrm{X}$ infection, $1-R R_{n O m-O m}^{i}(X)$. Contact rate is assumed to be twice of that of the Omicron wave.

30 Legend abbreviations: D614G: individuals who only experienced one D614G infection; Beta: individuals who only experienced one Beta infection; Delta: individuals who only experienced one Delta infection; Omicron: individuals who only experienced one Omicron infection; Others: individuals who only experienced one SARS-CoV-2 infection with genotype other than the D614G, Beta, Delta and Omicron variants; Vacc: individuals who had received at least one dose of vaccines but had not yet been infected by SARS-CoV-2; Vacc-Omicron: individuals who were vaccinated first then infected by Omicron; D614G-Omicron: individuals who were first infected by D614G then infected by Omicron; Beta-Omicron: individuals who were infected by Beta first then infected by Omicron; Delta- 
medRxiv preprint doi: https://doi.org/10.1101/2022.02.11.22270854; this version posted February 13, 2022. The copyright holder for this preprint

(which was not certified by peer review) is the author/funder, who has granted medRxiv a license to display the preprint in perpetuity.

It is made available under a CC-BY-NC-ND 4.0 International license .

Omicron: individuals who were infected by Delta first then infected by Omicron; Others-Omicron: individuals who were infected by a variant other than D614G, Beta, Delta and Omicron first then infected by Omicron; Repeat exposures: individuals who were exposed to SARS-CoV-2 antigens more than once (through vaccination or infection) without Omicron infection; Repeat exposures-Omicron: individuals who were exposed to SARS-CoV-2 antigens more than twice (through vaccination or infection) then infected by Omicron. 


\section{Materials and Methods}

\section{Cohort design}

We conducted a prospective household cohort study of SARS-CoV-2 transmission at an urban and a rural site in South Africa from July 2020 to August 2021(4). The rural site was in Agincourt, a rural community in Mpumalanga Province, which has been a longstanding health and socio-demographic surveillance system site. The urban site was in Klerksdorp, an urban community located in the North West Province. This study was built upon the larger multiyear Prospective Household cohort study of Influenza, Respiratory Syncytial virus and other respiratory pathogens community burden and Transmission dynamics (PHIRST), which was conducted from 2016 to 2018 to monitor transmission of respiratory pathogens (55). The study was repurposed for SARS-CoV-2 during the pandemic. To study infection and reinfection with SARS-CoV-2, a total of 222 households (114 in the rural site and 108 in the urban site) with at least 3 household members were enrolled between July 2020 and August 2021, consisting of 638 and 557 participants in the rural and urban site, respectively. In the rural site, we first approached households from the 2017 and 2018 cohorts, and in the urban site, those from the 2016, 2017 and 2018 cohorts. To supplement the sample size of the cohorts, we enrolled additional households at each site using the same methods as for the initial PHIRST study. Baseline demographic factors and information on underlying medical conditions were collected at enrollment (Table 1). Throughout the study period, household members were visited twice a week by study nurses and trained lay fieldworkders for collection of biological and clinical data. At each visit, upper respiratory tract specimens were collected using mid-turbinate nasal swabs, irrespective of symptom presentation. Data on symptoms, healthcare seeking behavior, hospitalization and death were captured at each follow up visit on a REDCap tablet-based real-time database. Respiratory specimens were tested by rRT-PCR for SARS-CoV-2. The lineage types of positive rRT-PCR specimens were determined by variant-specific rRT-PCR assay. Sera were collected at enrollment and approximately every 2 months during the 11-month follow-up period from all participants (see Figure 1 for timeline) and tested for the presence of SARS-CoV-2 antibodies.

\section{Laboratory methods}

The detailed laboratory methods were previously described (55). To briefly summarize, nucleic acids for real-time reverse transcription polymerase chain reaction (rRT-PCR) tests were extracted using the Hamilton Microlab NIMBUS Instrument (Hamilton, Nevada, USA) with the STARMag Universal Cartridge kit (Hamilton, Nevada, USA) and the STARMag Universal Cartridge kit (Seegene Inc., Seoul, Korea) according to the manufacturer's instructions. Specimens were tested for the presence of SARS-CoV-2 by rRT-PCR using the Seegene Allplex ${ }^{\text {TM }}$ 2019-nCoV kit (Seegene Inc., Seoul, Korea). Initial positive specimens were re-extracted and tested again. Only specimens with at least two out of three gene targets confirmed positive during the second test were considered as positive specimens. The cycle threshold $(\mathrm{Ct})$ value of each rRT-PCR test was recorded for further analysis. SARS-CoV-2 lineage was determined through Seegene variant I and II typing assays which differentiate variants Alpha (B.1.1.7), Beta (B.1.351), Delta (B1.617.2) and Gamma (P.1) (4).

Serum specimens were collected using venous blood, centrifuged into serum separator tubes and stored refrigerated immediately and transported to NICD. Aliquots of prespecified volume according to manufacturer instructions 
were tested for the presence of SARS-CoV-2 antibodies by the Roche Elecsys Anti-SARS-CoV-2 assay against nucleocapsid $(\mathrm{N})$ antigen (56). Assay readout above or equal to cutoff index 1 is considered as sero-positive, while below cutoff index 1 is considered as sero-negative. Negative control validations were performed using serum specimens from participants at both study sites prior to 2020 (4). An independent study benchmarking performances of available commercial and laboratory serologic assays demonstrated that the Roche Elecsys Anti-SARS-CoV-2 assay had high sensitivity and specificity across a wide range of severity spectrum for at least 6 months post-infection (57).

\section{Statistical analysis}

\subsection{Seroconversion and vaccination.}

Combining the longitudinal rRT-PCR assays and serological test results from the two study sites, we found that the seroconversion rate was $97.6 \%$ (583/600) among primary infection episodes with at least one blood specimen collected 30 days after their first rRT-PCR positive test. Among 639 individuals with negative serological specimen(s) after their first blood specimen was taken but who seroconverted later, 86\% (549/639) were confirmed by rRT-PCR during the study period, suggesting that twice-weekly rRT-PCR testing captured most of the infections during the study period. Among 706 seroconverted individuals with at least one follow-up blood specimens after seroconversion, only $40(5.7 \%)$ later sero-reverted (sero-reversion is defined as sero-converted individual who became sero-negative the subsequent serologic test). The baseline characteristics of all individuals are reported in Table 1. 10\% individuals received at least one dose of SARS-CoV-2 vaccine and 5\% were fully vaccinated within the study period of PHIRSTC. We censored individuals after their vaccination episode.

\subsection{Defining and typing variant-specific infection episodes.}

For each individual in the study, we defined the duration of a SARS-CoV-2 infection episode as the time interval between the first and the last of a set of positive rRT-PCR test, where consecutive positive tests were separated by less than 30 days. If at least one positive rRT-PCR test within the infection episodes was identified as any of the variants of concern (VOC) (defined by the WHO) Alpha (B.1.1.7), Beta (B.1.351), Delta (B.1.617.2), the lineage of the infection episode was assigned to the identified VOC. If all lineage-typed rRT-PCR tests within an infection episode were identified as the D614G, then the lineage of the infection episode was assigned to D614G. If none of the positive rRT-PCR specimens within an infection episode had a lineage defined, the lineage of the infection episode was labeled as inconclusive. In total, we observed 669 infection episodes, including 634/669 (95\%) with defined lineages. For the 35/669 (5\%) infection episodes with inconclusive lineages, we assigned the infection to the dominant SARS-CoV-2 lineage at the study site identified within the month of the infection episode. After lineage extrapolation, $108 / 669(16 \%)$ of the infection episodes were estimated to be caused by D614G lineage; 245/669 (37\%) infection episodes were estimated to be caused by VOC Beta lineage; 299/669 (45\%) infection episodes were estimated to be caused by VOC Delta lineage; 17/669 (3\%) were estimated to be caused by other lineages.

\subsection{Characterizing the relative viral RNA shedding kinetics of SARS-CoV-2 infection episodes.}


When interpreting Ct values from rRT-PCR test by the Seegene Allplex ${ }^{\mathrm{TM}}$ 2019-nCoV kit, it is important to stress that the $\mathrm{Ct}$ value of a single rRT-PCR test is not a direct measurement of the quantity of viral genetic material present in an individual specimen (in absolute terms) (58). Many factors could influence the Ct value of a rRT-PCR test, including but not limited to the specimen quality, extraction method, chemistry of reagents, gene targets. Further, $\mathrm{Ct}$ values cannot be directly compared between assays of different types (59). However, comparing serial Ct values and/or $\mathrm{Ct}$ values of different groups of population collectively, based on rRT-PCR tests from the same assay and laboratory setting, does reflect the relative variation in terms of viral genetic material concentration over time and between population subgroups $(58,59)$.

We measure the viral RNA shedding intensity of a given specimen as the differences between the cycle threshold value of Seegene Allplex ${ }^{\mathrm{TM}} 2019-\mathrm{nCoV}$ kit's detection limit $\left(C t_{\text {limit }}=40\right)$ to the $\mathrm{N}$ gene target $\mathrm{Ct}$ value of the specimen, i.e. $d C t_{\text {specimen }}^{N}=C t_{\text {limit }}-C t_{\text {specimen }}^{N}$. For the $\mathrm{Ct}$ value, we are only considering the $\mathrm{N}$ gene target to avoid between-target variation. In this study, instead of directly interpreting the Ct value of a single rRT-PCR test, we fit a mathematical model to capture the temporal kinetics of $d C t$ for longitudinally collected specimens and extract statistical summaries of RNA shedding intensity for each infection episode. In particular, following the method presented in (60), for each infection episode, we modelled the $d C t$ kinetics during the "RNA proliferation stage", characterized by linear growth in $d C t$ until a peak in $d C t$ is reached; and during the "RNA clearance stage", characterized by linear decline (in $d C t$ ) from the peak until the last rRT-PCR positive result. The duration of the "RNA proliferation stage" $\tau_{p}$ was defined as the time from $d C t$ first exceeding the detection threshold (i.e., $R L_{\text {specimen }}^{N}>$ 0 , or $\left.C t_{\text {specimen }}^{N}<40\right)$ until the peak of $d C t$; the duration of the "RNA clearance stage" $\tau_{c}$ was defined as the time from peak $d C t$ to $\mathrm{Ct}$ dropping below detection threshold (i.e., $C t_{\text {specimen }}^{N} \geq 40$ ). The duration of rRT-PCR positivity $\tau_{s}$ for each infection episode was defined as the total duration of the RNA proliferation and RNA clearance stages: $\tau_{s}=\tau_{p}+\tau_{c}$. The duration of RNA proliferation $\tau_{p}$, RNA clearance $\tau_{c}$, rRT-PCR positivity $\tau_{s}$, as well as the peak shedding intensity $d C t_{\text {specimen }}^{N}$ were estimated for each infection episodes using Markov Chain Monte Carlo (MCMC) method.

The fit of the RNA shedding trajectories for each variant are shown in Figure 2A-G. In addition, we performed multivariable regression analysis to evaluate the dependencies of duration of rRT-PCR positivity and peak $d C t$ on participants' characteristics including age, sex, BMI, HIV infection status, symptom status, variant type, and evidence of prior infection. Since the nasal swab sampling period ended on August 28, 2021, around the peak of the Delta wave in both sites, we limit our analysis to infection episodes with first positive PCR sample 30 days prior to the end of sampling to avoid censoring bias. The result of the regression is presented in Figure 2H-I.

\subsection{Assigning the lineage of prior infections among seropositive individuals.}

For individuals who seroconverted without a rRT-PCR confirmed infection episode (prior to the start of the PHIRSTC cohort), we assigned the lineage type of the individual's unobserved infection according to lineages' prevalence

(based on the infection episodes that had lineage information) at the study site in the month of the earliest seropositive specimen. 


\subsection{Risk factors associated with SARS-CoV-2 (re)infections.}

For each individual in the urban and rural sites, we first reconstructed the status of prior and ongoing infections at a daily time resolution, based on the lineage-typed infection episodes and serologic results described in Section 3.3 and 3.4 above. Specifically, we denoted a person-day observation of individual $i$ 's infection status at day $t$ as $O_{i}^{t}$. We set $O_{i}^{t}=1$ if individual $i$ acquired infection on day $t$ (i.e. the date of viral acquisition, marking the start of an infection episode), and set $O_{i}^{t}=0$ if individual $i$ didn't acquire infection on day $t$. Individuals without rRT-PCR positive results throughout the study period were assumed to escape infection throughout the observation period $\left(O_{i}^{t}=0\right.$, for all $\left.\mathrm{t}\right)$. For individuals with observed infection episodes during the study period, the individual $\mathrm{Ct}$ kinetics were modelled as in Section 3.3. The model allowed us to estimate the time at which $\mathrm{Ct}$ crossed the rRT-PCR detection limit $(C t=$ $40)$. We assumed that the time $t_{\text {inf }}$ of acquiring infection $\left(O_{i}^{t_{\text {inf }}}=1\right)$ occurred $\delta T_{\text {cryptic }}=4$ days prior to the $\mathrm{Ct}$ crossing the detection threshold (61). The time period from infection onset to viral RNA clearance (i.e., the estimated time of Ct crossing below rRT-PCR detection limit at cycle threshold of 40) was considered as a period of active infection, when an individual cannot get infected again, thus this refractory period was excluded from the regression analysis of (re)infection risk.

After having reconstructed the daily infection status for each individual, we modeled the daily probability of (re)infection for each individual using logistic regression. The risk factors considered in the regression analysis included the individual's age (allowing for variant-specific age effects), sex, body mass index (BMI), HIV infection status, household size, household crowding, variant type, study site, time since prior infection, SARS-CoV-2 exposure intensity from household members with on-going SARS-CoV-2 infection, and SARS-CoV-2 prevalence in the community. We included household and individual level hierarchical random effects in the regression model. We estimated that the SARS-CoV-2 exposure intensity from household members with on-going SARS-CoV-2 infections at time $t$ as the sum of the estimated $d C t \mathrm{~s}$ of all infected household members (62). The $d C t$ of household member $j$ at time $t$ was measured as $\mathrm{d} C t_{j}^{t}=C t_{\text {limit }}-C t_{j}^{t}$. Thus the "force of infection" $\lambda_{i}^{t}$ exerted on individual $i$ at time $t$ can be expressed as $\lambda_{i}^{t} \propto \sum_{j \neq i} \mathrm{~d} C t_{j}^{t}$, where $j$ is the sum over all infected household members at time $t$. We further discriminated the "force of infection" during the RNA proliferation and RNA clearance phases of infections in household members. We estimated the weekly SARS-CoV-2 community prevalence as the prevalence of each variant in the entire study site (no of variant-specific infections that week/population tested that week). Individual and household level random effects were also included in the logistic regression.

Since the nasal swab sampling period ended on August 28, 2021, around the peak of the Delta wave in both sites, we limit our analysis to infection episodes with first positive PCR sample 30 days prior to the end of sampling to avoid censoring bias. In total, 21\% (303407 person-days/1472400 person days) of the total person-days of observation were excluded from the regression during the entire study period due to missing nasal swab visits, missing serologic status, or individuals experiencing an active infection episode. A household with an individual with chronical SARS-CoV-2 infection was also excluded. The results of the regression are presented in Figure 2J. 
In this Section, we use PHIRST-C's urban site as a sentinel for SARS-CoV-2 antigen exposure(s) history (including both infection and vaccination) for the broader district where the study is located (Dr Kenneth Kaunda District). We reconstruct the population-level prevalence of different type of antigen exposures over time before Omicron and use this information as the initial condition in models that project the impact of Omicron. We focus on the urban site (Klerksdorp) as it is a major city of the Dr Kenneth Kaunda District.

4.1 Estimating the reporting rates of D614G, Beta, and Delta during the first three epidemic waves in the Dr Kenneth Kaunda District.

Here we compare the carefully monitored weekly PHIRST-C infection data with weekly surveillance data for the broader district to estimate aspects of SARS-CoV-2 dynamics in the district, including the rate of under-reporting for each of the pre-Omicron variants, pre-Omicron infection histories, and the impact of the Omicron wave. The dark blue bars in Figure 3F top panel shows the weekly incidence $I_{\text {case }}(t)$ of SARS-CoV-2 cases in the Dr Kenneth Kaunda District, North West Province, South Africa during the first three epidemic waves captured by SARS-CoV-2 surveillance. We calculate the weekly cumulative case rate $C_{\text {case }}(t)$ at time $t$ as $C_{\text {case }}(t)=\sum_{\tau=\text { week 12,2020 }}^{t} I_{\text {case }}(\tau)$, where week 12, 20202 is the week when the district starts reporting SARS-CoV-2 cases. We denote the cumulative infection attack rate at time $t$ (proportion of the population infected by each of these variants at time $\mathrm{t}$ ) for D614G, Beta, Delta (and others) variant as $C_{\text {infection }}^{D 614 G}(t), C_{\text {infection }}^{\text {Beta }}(t)$, and $C_{\text {infection }}^{\text {Delta \& Others }}(t)$ respectively. We estimate $C_{\text {infection }}^{D 614 G}(t), C_{\text {infection }}^{\text {Beta }}(t)$, and $C_{\text {infection }}^{\text {Delta } \text { Others }}(t)$ within the time period of the PHIRST-C cohort based on PHIRSTC's serology and variant-typed SARS-CoV-2 infection episodes (Detailed in Materials and Methods Section 3). We denote the reporting rate of $\mathrm{D} 614 \mathrm{G}$, Beta, and Delta (equal to Others) in the urban site as $\beta_{D 614 G}, \beta_{B e t a}$, and $\beta_{\text {Delta \& others. }}$. We assume that the reporting rate for each variant (within each epidemic wave) is constant and that Other variants (low frequency, mostly in between second and the third waves. See Figure 1F) have the same reporting rate as Delta. The cumulative case rate $C_{\text {case }}(t)$ and cumulative infection rates $C_{\text {infection }}^{D 614 G}(t), C_{\text {infection }}^{\text {Beta }}(t)$, and $C_{\text {infection }}^{\text {Delta } \text { Others }}(t)$ at time $t$ satisfy $C_{\text {case }}(t)=\beta_{D 614 G} \times C_{\text {infection }}^{\text {D614G }}(t)+\beta_{\text {Beta }} \times C_{\text {infection }}^{\text {Beta }}(t)+\beta_{\text {Delta \& others }}$. Given $C_{\text {case }}(t), C_{\text {infection }}^{\text {D614G }}(t), C_{\text {infection }}^{\text {Beta }}(t)$, and $C_{\text {infection }}^{\text {Delta } \& \text { others }}(t)$ for $t$ within the PHIRST-C cohort, we can estimate $\beta_{D 614 G}, \beta_{\text {Beta }}$, and $\beta_{\text {Delta } \& \text { others }}$ using linear regression without intercept. The regression analysis was performed using R package lme4 version 1.1-27.1 (63).The estimates are reported in Table S1.

Table S1: Reporting rate for each variant during the first three epidemic waves in Dr Kenneth Kaunda district, estimated by comparing infections in the PHIRST study with passive surveillance data in the broader district.

\begin{tabular}{|l|l|l|}
\hline Parameter & Estimate & $\mathbf{9 5 \%}$ CI \\
\hline$\beta_{D 614 G}$ & 0.036 & $0.035-0.038$ \\
\hline$\beta_{\text {Beta }}$ & 0.033 & $0.030-0.036$ \\
\hline$\beta_{\text {Delta \& others }}$ & 0.094 & $0.087-0.010$ \\
\hline
\end{tabular}


4.2 Estimating the weekly incidence rate of SARS-CoV-2 infection based on case incidence and reporting rate in Dr Kenneth Kaunda

Based on the variant-specific reporting rate $\beta_{X}(X \in\{D 614 G$, Beta, Delta \& Others $\})$ estimated in Section 4.1 and the variants' proportions $p_{X}(t)$ at a given time point $t$, we can express the overall reporting rate $\beta_{\text {overall }}(t)$ at time $t$ as:

$$
\beta_{\text {overall }}(t)=\sum_{X} \beta_{X} \times p_{X}(t)=I_{\text {case }}(t) / I_{\text {infection }}(t)
$$

Where $I_{\text {case }}(t)$ is the weekly incidence rate of SARS-CoV-2 cases reported to the Dr Kenneth Kaunda District at time $t$ and $I_{\text {infection }}(t)$ is the weekly incidence rate of SARS-CoV-2 infections. We can thus estimate $I_{\text {infection }}(t)$ as

$$
I_{\text {infection }}(t)=I_{\text {case }}(t) / \beta_{\text {overall }}(t)
$$

The estimated $I_{\text {infection }}(t)$ prior to the end of PHIRST-C (September 2021) is visualized in Figure 3F top panel (light blue bars prior to September 2021).

\subsection{Reconstructing the SARS-CoV-2 antigen exposure history in Dr Kenneth Kaunda by September 2021.}

In addition to allowing to trace SARS-CoV-2 infection history in detail, the PHIRST-C cohort also recorded participants' timing of vaccinations. Specifically, at time $t$, we denote the proportion of the PHIRST-C urban site population with a single prior SARS-CoV-2 infection, which is to D614G, as $p_{D 614 G}^{\text {expo }}(t)$; with $p_{\text {Beta }}^{\text {expo }}(t)$ and $p_{\text {Delta }}^{\text {expo }}(t)$ representing the same quantities for Beta and Delta. We denote past vaccination (at least one dose) as $p_{\text {Vacc }}^{\text {expo }}(t)$; with repeat exposures (including repeat infections, vaccination followed by infection, or infection followed by vaccination) as $p_{R E}^{\text {expo }}(t)$. Then we can express the proportion of population with past SARS-CoV-2 infections at time $t$ as:

$$
p_{\text {infection }(s)}^{\text {expo }}(t)=p_{D 614 G}^{\text {expo }}(t)+p_{\text {Beta }}^{\text {expo }}(t)+p_{\text {Delta }}^{\text {expo }}(t)+p_{R E}^{\text {expo }}(t)
$$

If we denote the cumulative infection attack rate in Dr Kenneth Kaunda $C_{\text {infection }}(t)=\sum_{\tau=0}^{t} I_{\text {infection }}(\tau)$ and assume the PHIRST-C urban site is a representative survey of the population in Dr Kenneth Kaunda, we can express the cumulative prevalence of past exposure of a given exposure type $X$ at time $t$ as:

$$
C_{X}(t)=C_{\text {infection }}(t) \times p_{X}^{\text {expo }} / p_{\text {infection }(s)}^{\text {expo }}
$$

Where $X \in\{D 614 G$, Beta,Delta, Repeat Exposures,Vaccinated $\}$. In Figure S1 (below) and Figure 3F bottom panel, we visualize the proportion of population with a specific SARS-CoV-2 antigen exposure history.

\section{Modelling the transmission dynamics of the Delta and Omicron variant from September 2021 to the end of}

\section{Omicron wave}

\subsection{Overview of protective immunity}

To project the impact of Omicron, we need to model how immunity from infection with pre-Omicron variants and/or vaccination will impact the probabilities of infection, transmission and severe outcomes. The effectiveness of protective immunity $(I E)$ can be measured very broadly as $I E=1-R R$ where $R R$ is the relative risk of an outcome of interest (infection, transmission, hospitalization, etc.) and the comparison is against individuals with no prior 
immunity or with different types of immunity. In particular, we consider three aspects of protection from prior infection/vaccination:

1) Prior infection/vaccination could reduce the host's susceptibility to reinfection $I E^{i}$, measured as $I E^{i}=1-$ $R R^{i}$ where $R R^{i}$ is the relative risk of reinfection/breakthrough infection when compared to the infection risk in a naïve population, controlling for same level of exposure.

2) Prior infection/vaccination could reduce the risk of onward transmission given reinfection/breakthrough infection $I E^{t \mid i}$, measured as $I E^{t \mid i}=1-R R^{t \mid i}$ where $R R^{t \mid i}$ is the relative risk of onward transmission for reinfections/breakthrough infection when compared to that of primary infections, conditional on the same contact rate. Here $R R^{t \mid i}=R R^{t d \mid i} \times R^{t s \mid i}$ can be further broken down into the product of reduction in the duration and intensity of shedding $\left(R R^{t d \mid i}\right.$ and $\left.R R^{t s \mid i}\right)$.

3) Prior infection/vaccination could reduce the risk of disease given reinfection/breakthrough infection. The concept of COVID-19 disease is generic and could encompass a wide spectrum of severity endpoint including symptomatic cases, hospitalizations, and deaths. In this study, we used symptomatic illness as the severity end point. The effectiveness of protective immunity against being symptomatic case, conditional on infection can be measured as $I E^{c \mid i}=1-R R^{c \mid i}$, where $R R^{c \mid i}$ is the relative risk of disease for reinfections/breakthrough infections compared to primary infections.

\subsection{Modelling boosting and evasion of protective immunity in the Omicron era}

Repeat exposures to SARS-CoV-2 antigens can result from repeated infections, booster shots following primary vaccine schedule(s), infection following vaccination or vice versa. Multiple exposures could stimulate a recall response and boost the level of protective immune effectiveness $I E$ through reducing $R R$ further from the baseline protective immunity provided by primary exposure. For example, let's denote the protective immunity conferred by primary infection with a pre-Omicron $(\mathrm{pOm})$ strain as $I E_{p o m}(p O m)=1-R R_{p o m}(p O m)$. We can express the level of protective immunity conferred by reinfection with pre-Omicron strains as $I E_{\text {pom-pom }}(p O m)=1-$ reinfection with a pre-Omicron strain. For $R R_{\text {pom }}(p O m)<1$ ), the larger $\rho_{\text {pom }}$, the greater the reduction $R R_{\text {pom-pom }}(p O m)$ when compared to $R R_{\text {pom }}(p O m)$, and the higher the protection conferred by boosting.

On the other hand, in face of an antigenically distinct variant like Omicron, prior immunity may not be as efficacious due to reduced ability to recognize the antigen through immune memory, leading to elevation in $R R$ and consequently reduction in $I E$. For example if we denote the protective immunity conferred by primary infection with a pre-Omicron strain against refection by pre-Omicron as $I E_{p o m}(p O m)=1-R R_{p o m}(p O m)$, we can express the level of protective immunity conferred by pre-Omicron strain infection against Omicron $(\mathrm{Om})$ as $I E_{\text {pom }}(\mathrm{Om})=1-R R_{\text {pom }}(\mathrm{Om})=$ $1-\left(R R_{p o m}(p O m)\right)^{1-\sigma_{O m}}$, where $\sigma_{O m}$ measures the degree of immune evasion by Omicron. When $\sigma_{O m}=0$, $I E_{\text {pom }}(O m)=I E_{\text {pom }}(p O m)$, indicating no immune escape. When $\sigma_{O m}=1, I E_{p o m}(O m)=0$, indicating $100 \%$ immune escape. We can further model the combined effects of boosting and immune escape. For example, if we 
consider an individual first infected with a pre-Omicron strain, followed by an Omicron infection, we can express the individual's level of protective immunity against further Omicron infection as $I E_{\text {pom-Om }}(O m)=1-$ $R R_{\text {pom-Om }}(O m)=1-\left(R R_{p o m}(p O m)\right)^{1-\sigma_{O m}+\rho_{O m}}$.

\subsection{Notation conventions and an exhaustive list of different types of protective immunity considered.}

Following Section 5.1 and 5.2, we use $1-R R$ as a measurement of the level of protective immunity. In particular, $R R=R R_{Y}^{X}(S)$ can be broken down into three independent dimensions: 1) the type of protective immunity $X$ (superscript), 2) the antigen exposure history $Y$ that confers immunity (subscript), 3) the viral strain $S$ against which immunity is directed (brackets).

Where $X \in\{i, t|i, t d| i, t s|i, h| i\}$ and:

- $\quad i$ denotes protection in terms of susceptibility against infection.

- $t \mid i$ denotes protection against transmission given infection.

- $t d \mid i$ denotes protection against transmission through reduced duration of shedding, given infection.

- $t s \mid i$ denotes protection against transmission through reduced intensity of shedding, given infection.

- $\quad h \mid i$ denotes protection against hospital admission given infection.

Where $Y \in\{p O m, O m, p O m-p O m, p O m-O m\}$ and:

- $\quad$ Om denotes immune histories including only one antigen exposure by either pre-Omicron (including D614G, Alpha, Beta, Delta, and other non-Omicron variants in South Africa) infection or a primary schedule of vaccination.

- Om denotes immune histories consisting in a single exposure to SARS-CoV-2 via an Omicron infection.

- $\quad p O m-p O m$ denotes immune histories including at least two antigen exposures with first exposure being either pre-Omicron strain or vaccination and second exposure either pre-Omicron strain or vaccination.

- $\quad p O m-O m$ denotes immune history including at least two antigen exposures with the first exposure being either pre-Omicron strain or vaccination and the second exposure being an Omicron infection.

Where $S \in\{p O m, O m\}$ and:

- $\quad p O m$ denotes protection against pre-Omicron strains.

- $O m$ denotes protection against Omicron.

In this study, since the antigenic difference between Omicron and all previously circulating strains (D614G, Alpha, Beta, Delta) is much larger than antigenic differences among previously circulating strains (16), we do not differentiate immunity between pre-Omicron variants (all pre-Omicron variants confer the same type of homologous and heterologous immunity to each other, and we also consider the vaccine to be antigenically similar to pre-Omicron strains since it is based on wild type strain). For simplicity, considering the low vaccination rate in South Africa, we assume that a full vaccination schedule confers similar levels of protection as an infection with a pre-Omicron strain (i.e., D614G, Alpha, Beta, Delta). In reality, a full schedule of vaccination likely confers lower level of protection against infection over long time scales due to waning, irrespective of the vaccine platforms $(17,23,30)$. Differences 
in long-term protection against onward transmission and hospitalization remain unclear. For simplicity and given the relatively short time scale being considered, we also assume that the first two antigen exposures dominate the acquisition of immunity and subsequent infections by Omicron beyond the first two may not change immune memory against Omicron (15). An exhaustive list of all immunity protection scenarios considered in this study is shown in

Table S2.

Table S2: List of the definitions of different types of protective immunity.

\begin{tabular}{|c|c|c|c|}
\hline Variable name & Definition & Value/Expression & Notes \& Reference \\
\hline$R R_{\text {pom }}^{i}(\mathrm{pOm})$ & $\begin{array}{l}\text { Relative risk of infection by a pre-Omicron variant } \\
\text { among individuals previously infected by a pre- } \\
\text { Omicron variant, or in vaccinated individuals } \\
\text { (breakthrough infection), compared to naïve } \\
\text { individuals, and conditional on the same contacts. }\end{array}$ & 0.12 & Estimated from this study. \\
\hline$R R_{\text {pOm }}^{t \mid i}(p O m)$ & $\begin{array}{l}\text { Relative risk of onward transmission of pre- } \\
\text { Omicron variants among individuals infected with } \\
\text { pre-Omicron variant for the second time, or in } \\
\text { vaccinated individuals (breakthrough infection), } \\
\text { compared to naïve individuals, and conditional on } \\
\text { infection. }\end{array}$ & 0.4 & (24) \\
\hline$\sigma_{O m}^{i}$ & $\begin{array}{l}\text { Degree of immune evasion with respect to } \\
\text { susceptibility to infection by the Omicron variant. }\end{array}$ & $\mathrm{NA}$ & $\begin{array}{l}\text { Free parameter to be } \\
\text { explored in this study } \\
\text { (Figure } 4 \mathrm{~A} \text { ). See also } \\
\text { expression for } R R_{\text {pOm }}^{i}(\mathrm{Om}) \text {. }\end{array}$ \\
\hline$\sigma_{O m}^{t \mid i}$ & $\begin{array}{l}\text { Degree of immune evasion with respect to } \\
\text { reduction in onward transmission by the Omicron } \\
\text { variant, conditional on infection. }\end{array}$ & $\mathrm{NA}$ & $\begin{array}{l}\text { Free parameter to be } \\
\text { explored in this study } \\
\text { (Figure 4A). }\end{array}$ \\
\hline$\rho_{\text {pOm }}^{i}$ & $\begin{array}{l}\text { Degree of boosted immunity by pre-Omicron } \\
\text { infection/vaccination with respect to reducing } \\
\text { susceptibility to infection. }\end{array}$ & 0.5 & $\begin{array}{l}\text { Assumed, see also } \\
\text { expression for } \\
R R_{\text {pom-pom }}^{i}(p O m)\end{array}$ \\
\hline$\rho_{\text {pom }}^{t \mid i}$ & $\begin{array}{l}\text { Degree of boosted immunity by pre-Omicron } \\
\text { infection/vaccination with respect to reduction in } \\
\text { onward transmission. }\end{array}$ & 0.5 & Assumed. \\
\hline$\rho_{\text {pom }}^{h \mid i}$ & $\begin{array}{l}\text { Degree of boosted immunity by pre-Omicron } \\
\text { infection/vaccination with respect to reduction in } \\
\text { hospital admission. }\end{array}$ & 0.5 & Assumed. \\
\hline$\rho_{O m}^{i}$ & $\begin{array}{l}\text { Degree of boosted immunity by Omicron } \\
\text { infection/vaccination with respect to reduction in } \\
\text { susceptibility to infection. }\end{array}$ & NA & $\begin{array}{l}\text { Free parameter to be } \\
\text { explored in this study } \\
\text { (Figure 4D). }\end{array}$ \\
\hline$\rho_{O m}^{t \mid i}$ & $\begin{array}{l}\text { Degree of boosted immunity by Omicron } \\
\text { infection/vaccination with respect to reduction in } \\
\text { onward transmission. }\end{array}$ & NA & $\begin{array}{l}\text { Free parameter to be } \\
\text { explored in this study } \\
\text { (Figure 4D). }\end{array}$ \\
\hline$R R_{\text {pom }}^{i}(\mathrm{Om})$ & $\begin{array}{l}\text { Relative risk of infection by Omicron strain } \\
\text { between population with prior infection by pre- } \\
\text { Omicron strain/full schedule of vaccination* and }\end{array}$ & $\left(R R_{p O m}^{i}(p O m)\right)^{1-\sigma_{O m}^{i}}$ & \\
\hline
\end{tabular}




\begin{tabular}{|c|c|c|c|}
\hline & $\begin{array}{l}\text { naïve population, conditional on the same } \\
\text { exposure. }\end{array}$ & & \\
\hline$R R_{p O m}^{t \mid i}(O m)$ & $\begin{array}{l}\text { Relative risk of onward transmission by Omicron } \\
\text { strain (given infection) between population with } \\
\text { prior infection by pre-Omicron strain/full schedule } \\
\text { of vaccination* and naïve population, conditional } \\
\text { on infection. }\end{array}$ & $\left(R R_{\text {pom }}^{t \mid i}(p O m)\right)^{1-\sigma_{O m}^{t \mid i}}$ & \\
\hline$R R_{\text {pOm-pom }}^{i}(p O m)$ & $\begin{array}{l}\text { Relative risk of infection by a pre-Omicron strain } \\
\text { in individuals with more than one prior } \\
\text { infection/vaccination* (both primary and } \\
\text { secondary infections are pre-Omicron), compared } \\
\text { to naïve individuals. }\end{array}$ & $\left(R R_{\text {pom }}^{i}(p O m)\right)^{1+\rho_{\text {pom }}^{i}}$ & \\
\hline$R R_{\text {pOm-pom }}^{t \mid i}(p O m)$ & $\begin{array}{l}\text { Relative risk of onward transmission (given } \\
\text { infection by a pre-Omicron strain) in individuals } \\
\text { with more than one prior infection/vaccination* } \\
\text { (both primary and secondary infections are pre- } \\
\text { Omicron), compared to naïve individuals. }\end{array}$ & $\left(R R_{\text {pom }}^{t \mid i}(p O m)\right)^{1+\rho_{\text {pom }}^{t \mid i}}$ & \\
\hline$R R_{\text {pom-pom }}^{i}(\mathrm{Om})$ & $\begin{array}{l}\text { Relative risk of infection by Omicron in } \\
\text { individuals with more than one prior } \\
\text { infection/vaccination* (both primary and } \\
\text { secondary infections are pre-Omicron), compared } \\
\text { to naïve individuals. }\end{array}$ & $\left(R R_{\text {pOm }}^{i}(p O m)\right)^{1+\rho_{\text {pOm }}^{i}-\sigma_{O m}^{i}}$ & \\
\hline$R R_{\text {pom-pom }}^{t \mid i}(O m)$ & $\begin{array}{l}\text { Relative risk of onward transmission (given } \\
\text { infection by Omicron) in individuals with more } \\
\text { than one prior infection/vaccination* (both } \\
\text { primary and secondary infections are pre- } \\
\text { Omicron), compared to naïve individuals. }\end{array}$ & $\left(R R_{\text {pom }}^{t \mid i}(p O m)\right)^{1+\rho_{\text {pOm }}^{t \mid i}-\sigma_{O m}^{t \mid i}}$ & \\
\hline$R R_{\text {pOm-Om }}^{i}(\mathrm{Om})$ & $\begin{array}{l}\text { Relative risk of infection by Omicron in } \\
\text { individuals with more than one prior } \\
\text { infection/vaccination* (with primary infection by a } \\
\text { pre-Omicron strain/vaccination and secondary } \\
\text { infection by Omicron), compared to naïve } \\
\text { individuals. }\end{array}$ & $\left(R R_{\text {pOm }}^{i}(p O m)\right)^{1-\sigma_{O m}^{i}+\rho_{O m}^{i}}$ & \\
\hline$R R_{\text {pOm-Om }}^{t \mid i}(\mathrm{Om})$ & $\begin{array}{l}\text { Relative risk of onward transmission (given } \\
\text { infection by Omicron) in individuals with more } \\
\text { than one prior infection/vaccination* (with } \\
\text { primary infection by a pre-Omicron } \\
\text { strain/vaccination and secondary infection by } \\
\text { Omicron), compared to naïve individuals. }\end{array}$ & $\left(R R_{\text {pOm }}^{t \mid i}(p O m)\right)^{1-\sigma_{O m}^{t \mid i}+\rho_{O m}^{t \mid i}}$ & \\
\hline$R R_{O m}^{i}(\mathrm{Om})$ & $\begin{array}{l}\text { Relative risk of infection by Omicron strain in } \\
\text { individuals with prior infection by Omicron, } \\
\text { compared to naïve individuals, conditional on the } \\
\text { same contacts. }\end{array}$ & $R R_{p O m}^{i}(p O m)$ & $\begin{array}{l}\text { Assuming similar level of } \\
\text { protection when compared to } \\
\text { pre-Omicron strains }\end{array}$ \\
\hline$R R_{O m}^{t \mid i}(O m)$ & $\begin{array}{l}\text { Relative risk of onward transmission (given } \\
\text { infection by Omicron) in individuals with prior }\end{array}$ & $R R_{p O m}^{t \mid i}(p O m)$ & $\begin{array}{l}\text { Assuming similar level of } \\
\text { protection when compared to } \\
\text { pre-Omicron strains }\end{array}$ \\
\hline
\end{tabular}




\begin{tabular}{|l|l|l|l|}
\hline & $\begin{array}{l}\text { infection with Omicron, compared to naïve } \\
\text { individuals. }\end{array}$ & & \\
\hline$R R_{O m-O m}^{i}(\mathrm{Om})$ & $\begin{array}{l}\text { Relative risk of infection by Omicron in individuals } \\
\text { with more than one prior infection (both primary } \\
\text { and secondary infections are with Omicron), } \\
\text { compared to naïve individuals. }\end{array}$ & $R R_{p O m-p o m}^{i}(\mathrm{pOm})$ & $\begin{array}{l}\text { Assuming similar level of } \\
\text { protection when compared to } \\
\text { pre-Omicron strains }\end{array}$ \\
\hline$R R_{O m-O m}^{t \mid i}(\mathrm{Om})$ & $\begin{array}{l}\text { Relative risk of onward transmission (given } \\
\text { infection by Omicron) in individuals with more than } \\
\text { one prior infection (both primary and secondary } \\
\text { infections are with Omicron), compared to naïve } \\
\text { individuals. }\end{array}$ & $R R_{p o m-p o m}^{t \mid i}(p O m)$ & $\begin{array}{l}\text { Assuming similar level of } \\
\text { protection when compared to } \\
\text { pre-Omicron strains }\end{array}$ \\
\hline$R R_{O m-O m}^{t \mid i}(\mathrm{Om})$ & $\begin{array}{l}\text { Relative risk of hospital admission (given infection } \\
\text { by Omicron) in individuals with more than one prior } \\
\text { infection (both primary and secondary infections } \\
\text { are with Omicron), compared to naïve individuals. }\end{array}$ & $R R_{p O m-p o m}^{t \mid i}(p O m)$ & $\begin{array}{l}\text { Assuming similar level of } \\
\text { protection when compared to } \\
\text { pre-Omicron strains }\end{array}$ \\
\hline
\end{tabular}

* For simplicity, given the low vaccination rate in South Africa, we assume that a full schedule of vaccination confers a similar level of protection as infection with pre-Omicron strains (i.e., D614G, Alpha, Beta, Delta). In reality, infection-induced immunity may confer superior protection against infection in the long run, relative to vaccination (17), while differences in magnitude and duration of protection against transmission and hospitalization remain unclear.

\subsection{Estimating the growth advantage of Omicron over Delta during its initial emergence.}

When Omicron was discovered in South Africa, the Delta epidemic had already declined and the Delta variant was circulating at low level in most locations (20). In Figure S2, the dots show the logarithmic of SARS-CoV-2 weekly incidence in the District of Kenneth Kaunda between weeks 35 and 48 in 2021. The epidemic curve can be viewed as the supposition of an exponential decay and an exponential growth, with the transition occurring around week 45 of 2021 (coinciding with the emergence of Omicron in South Africa). Here we assume that the exponential decay (prior to week 45, 2021) was driven by the Delta variant and the exponential growth (post week 45, 2021) was driven by the Omicron variant. We can thus model the epidemic curve as supposition of exponential decay and exponential growth, i.e.

$$
I_{\text {case }}(t)=A \times e^{\alpha t}+B \times e^{\beta t}
$$

Here $\alpha$ is the growth rate of Delta and $\beta$ is the growth rate of Omicron and $A$ and $B$ are the initial Incidence rate for Delta and Omicron when $t=0$. We fit this function to the observed epidemic curve between week 35 and week 48 using maximum likelihood method. We find a growth rate of -0.063 per day for the Delta variant (exponential decay) and 0.275 per day for the Omicron variant (exponential growth), indicating a growth advantage of 0.338 per day of Omicron over Delta in Dr Kenneth Kaunda District. Figure S2 shows the results of the fitting.

\subsection{State-space transmission model for Delta variant and projection of Delta spread from weeks 35 to 45,2021} history up to 3 repeat infections/immunizations. The equations governing the model are as follows: 


$$
\begin{gathered}
\frac{d s_{0}}{d t}=-\lambda_{0} s_{0}+\mu-\eta(t) s_{0}-v s_{0} \\
\frac{d i_{0}}{d t}=\lambda_{0} s_{0}-\gamma_{0}^{\text {Delta }} i_{0}-v i_{0}
\end{gathered}
$$

$$
\begin{gathered}
\frac{d i_{1}^{m}}{d t}=\lambda_{1}^{m} s_{1}^{m}-\gamma_{1}^{m} i_{1}^{m}-v i_{1}^{m} \\
\frac{d r_{1}^{m}}{d t}=\gamma_{1}^{m} i_{1}^{m}-\omega_{1} r_{1}^{m}-v r_{1}^{m} \\
\frac{d s_{2}}{d t}=-\lambda_{2} s_{2}+\sum_{m} \omega_{1} r_{1}^{m}+\sum_{m} \eta(t) s_{1}^{m}+\omega_{2} r_{2}-v s_{2}
\end{gathered}
$$

15

$$
\begin{aligned}
& \frac{d i_{2}}{d t}=\lambda_{2} s_{2}-\gamma_{2} i_{2}-v i_{2} \\
& \frac{d r_{2}}{d t}=\gamma_{2} i_{2}-\omega_{2} r_{2}-v r_{2}
\end{aligned}
$$

With the following expression for the force-of-infection:

$$
\begin{gathered}
\lambda_{0}=\alpha_{\text {scaling }} \times \beta_{\text {Delta }} \times(1+\Delta \beta \cos (2 \pi(t+\phi) / T)) \times\left(i_{0}+\sum_{m \in M} \epsilon_{1}^{m} i_{1}^{m}+\epsilon_{2} i_{2}\right) \\
\lambda_{1}^{m}=\theta_{1}^{m} \lambda_{0}
\end{gathered}
$$

25

$$
\lambda_{2}=\theta_{2} \lambda_{0}
$$

The infectious periods for $i_{1}^{m}$ and $i_{2}$ can be expressed as follows with respect to $i_{0}$ 's:

$$
\frac{1}{\gamma_{1}^{m}}=\frac{\kappa_{1}^{m}}{\gamma_{0}^{\text {Delta }}}
$$




$$
\frac{1}{\gamma_{2}}=\frac{\kappa_{2}}{\gamma_{0}^{\text {Delta }}}
$$

The definitions of the state variables are presented in Table S3, and the definitions of the model parameters are in Table S4. We initialized the model based on the reconstructed exposure history (Figure S1) by week 34. The only free parameter of the model was the rescaling factor on transmissibility $\alpha_{\text {scaling }}$ (Table S4). We optimized $\alpha_{\text {scaling }}$ so that the projected curve of new incidence would decay at a rate of -0.063 day $^{-1}$, matching the observation (Figure S2). The best fit for $\alpha_{\text {scaling }}$ was 0.44 . We further projected the SARS-CoV-2 antigen exposure history from week 35 to week 45 (prior to the emergence of the Omicron variant (Figure S3).

Table S3: State variables of the Delta variant compartmental transmission model.

\begin{tabular}{|c|c|c|}
\hline Variable name & Variable type & Definition \\
\hline$m \in M$ & Categorical & $\begin{array}{l}m \text { Indicates the type of primary SARS-CoV-2 antigen exposure from the set } M= \\
\{D 614 G, \text { Beta,Delta,Delta,Others, Vacc }\} \text { denotes immune history including only one antigen } \\
\text { exposure by pre-Omicron infection (including D614G, Beta, Delta, and other variants other than } \\
\text { Omicron ("Others") in South Africa) or a primary schedule of vaccination ("Vacc"). }\end{array}$ \\
\hline$s_{0}$ & State variable & $\begin{array}{l}\text { Fraction of susceptible in the population who are fully naïve against any SARS-CoV-2 infection and are } \\
\text { unvaccinated. }\end{array}$ \\
\hline$s_{1}^{m}$ & State variable & $\begin{array}{l}\text { Fraction of susceptible in the population who have experienced one infection or a full schedule of } \\
\text { vaccination, with } m \text { denoting the type of primary antigen exposure (i.e., variant type if infection or if } \\
\text { primed by vaccination). }\end{array}$ \\
\hline$S_{2}$ & State variable & $\begin{array}{l}\text { Fraction of susceptible in the population who have experienced two or more infections and } \\
\text { immunization combined. We assume that the first two immunizations will have the strongest impact on } \\
\text { the level of long-term protective immunity. }\end{array}$ \\
\hline$i_{0}$ & State variable & Fraction of population who got infected by Delta from population in $s_{0}$ \\
\hline$i_{1}^{m}$ & State variable & Fraction of population who got infected by Delta from population in $s_{1}^{m}$ \\
\hline$i_{2}$ & State variable & Fraction of population who got infected by Delta from population in $s_{2}$ \\
\hline$r_{0}$ & State variable & $\begin{array}{l}\text { Fraction of population who recovers from Delta in } i_{0} \text { compartment and enjoys a temporary period of full } \\
\text { immunity ( } 100 \% \text { protection) against any reinfection. }\end{array}$ \\
\hline$r_{1}^{m}$ & State variable & $\begin{array}{l}\text { Fraction of population who recovers from reinfection/breakthrough of Delta infection in } i_{1}^{m} \\
\text { compartment and enjoys a temporary period of full immunity ( } 100 \% \text { protection) against any reinfection. }\end{array}$ \\
\hline$r_{2}$ & State variable & $\begin{array}{l}\text { Fraction of population who recovers from further reinfection/breakthrough of Delta infection in the } i_{2} \\
\text { compartment and enjoys a temporary period of full immunity ( } 100 \% \text { protection) against any reinfection. }\end{array}$ \\
\hline
\end{tabular}

Table S4: Model parameters for the Delta variant transmission model.

\begin{tabular}{|c|c|c|c|}
\hline Parameter & Definition & Value & Notes/Reference \\
\hline$\mu$ & Annual birth rate in South Africa & 0.026 year $^{-1}$ & \\
\hline$v$ & Annual death rate in South Africa & 0.008 year $^{-1}$ & \\
\hline$\beta_{\text {Delta }}$ & Baseline transmission rate of Delta & 1.02 day $^{-1}$ & $\begin{array}{l}\text { Assuming that D614G has a basic reproduction } \\
\text { number of } 2 \text {, Alpha is } 1.7 \text { times more infectious } \\
\text { than D614G and Delta is } 1.5 \text { times more infectious }\end{array}$ \\
\hline
\end{tabular}




\begin{tabular}{|c|c|c|c|}
\hline & & & $\begin{array}{l}\text { than Alpha and D614G, Alpha, and Delta share the } \\
\text { same generation interval of } 1 \backslash \gamma_{0}^{\text {Delta }}=5 \text { days. }\end{array}$ \\
\hline$\Delta \beta$ & Magnitude of seasonal forcing & 0.15 & https://smw.ch/article/doi/smw.2020.20224 \\
\hline$\phi$ & Phase of seasonal forcing in South Africa & 180 days & $\begin{array}{l}\text { *South Africa locates in the Southern Hemisphere; } \\
\text { we assume a peak of seasonal transmission during } \\
\text { winter months }\end{array}$ \\
\hline$\theta_{1}^{m}$ & $\begin{array}{l}\text { Reduction in susceptibility to infection due } \\
\text { to protection from primary antigen } \\
\text { exposure }\end{array}$ & $R R_{\text {pOm }}^{i}(p O m)$ & See Table S2. \\
\hline$\theta_{2}$ & $\begin{array}{l}\text { Reduction in susceptibility to infection due } \\
\text { to protection from primary and secondary } \\
\text { antigen exposures }\end{array}$ & $R R_{\text {pom-pom }}^{i}(\mathrm{pOm})$ & See Table S2. \\
\hline$\alpha_{\text {scaling }}$ & $\begin{array}{l}\text { Transmission scaling factor to account for } \\
\text { factors such as non-pharmaceutical } \\
\text { interventions or heterogeneity in mixing } \\
\text { patterns }\end{array}$ & 0.44 (Fitted) & $\begin{array}{l}\text { Estimated for time period after week } 35 \text { of } 2021 \text {, } \\
\text { see Section } 5.6\end{array}$ \\
\hline $1 / \gamma_{0}^{\text {Delta }}$ & $\begin{array}{l}\text { Infectious period of Delta's primary } \\
\text { infection } i_{0}\end{array}$ & 5 days & $\begin{array}{l}\text { In the SIRS model, the generation interval is equal } \\
\text { to the infectious period. }\end{array}$ \\
\hline$\kappa_{1}^{m}$ & $\begin{array}{l}\text { Reduction in terms of duration of shedding } \\
\text { due to protection from primary antigen } \\
\text { exposure }\end{array}$ & $\left(R R_{\text {pom }}^{t \mid i}(p O m)\right)^{\frac{1}{2}}$ & $\begin{array}{l}\text { Assuming that reduction of onward transmission } \\
\text { (Table S2) is evenly split between reduction in } \\
\text { duration and intensity of shedding. }\end{array}$ \\
\hline $1 / \gamma_{1}^{m}$ & $\begin{array}{l}\text { Infectious period of Delta's first } \\
\text { reinfection/breakthrough of infection } i_{1}^{m}\end{array}$ & $\kappa_{1}^{m} / \gamma_{0}^{\text {Delta }}$ & NA \\
\hline$\kappa_{2}$ & $\begin{array}{l}\text { Reduction in terms of duration of shedding } \\
\text { due to protection from primary and } \\
\text { secondary antigen exposures }\end{array}$ & $\left(R R_{p O m-p O m}^{t \mid i}(p O m)\right)^{\frac{1}{2}}$ & $\begin{array}{l}\text { Assuming that reduction of onward transmission } \\
\text { (Table S2) is evenly split between reduction in } \\
\text { duration and intensity of shedding. }\end{array}$ \\
\hline $1 / \gamma_{2}$ & $\begin{array}{l}\text { Infectious period of Delta's second } \\
\text { reinfection/breakthrough infection } i_{2}\end{array}$ & $\kappa_{2} / \gamma_{0}^{\text {Delta }}$ & NA \\
\hline$\epsilon_{1}^{m}$ & $\begin{array}{l}\text { Reduction in terms of intensity of shedding } \\
\text { due to protection from primary antigen } \\
\text { exposures }\end{array}$ & $\left(R R_{\text {pom }}^{t \mid i}(p O m)\right)^{\frac{1}{2}}$ & $\begin{array}{l}\text { Assuming that reduction of onward transmission } \\
\text { (Table S2) is evenly split between reduction in } \\
\text { duration and intensity of shedding. }\end{array}$ \\
\hline$\epsilon_{2}$ & $\begin{array}{l}\text { Reduction in terms of intensity of shedding } \\
\text { due to protection from primary and } \\
\text { secondary antigen exposures }\end{array}$ & $\left(R R_{\text {pOm-pom }}^{t \mid i}(p O m)\right)^{\frac{1}{2}}$ & $\begin{array}{l}\text { Assuming that reduction of onward transmission } \\
\text { (Table S2) is evenly split between reduction in } \\
\text { duration and intensity of shedding. }\end{array}$ \\
\hline$\eta(t)$ & Vaccination rate over time & Time dependent & $\begin{array}{l}\text { Estimated based on the vaccination rate for } \\
\text { population in the PHIRST-C urban cohort. }\end{array}$ \\
\hline $1 / \omega_{0}$ & $\begin{array}{l}\text { Duration of period of full immunity } \\
\text { following primary infection with Delta }\end{array}$ & 60 days & Assumed \\
\hline $1 / \omega_{1}$ & $\begin{array}{l}\text { Duration of period of full immunity } \\
\text { following primary infection with Delta } \\
\text { following first reinfection with Delta }\end{array}$ & 60 days & Assumed \\
\hline $1 / \omega_{2}$ & $\begin{array}{l}\text { Duration of period of full immunity } \\
\text { following primary infection with Delta } \\
\text { following repeat reinfections with Delta }\end{array}$ & 60 days & Assumed \\
\hline$\Omega_{1}^{m}$ & $\begin{array}{l}\text { Operator that maps the primary exposure } \\
\text { (Delta infection/vaccination) to different }\end{array}$ & $\begin{array}{l}\Omega_{1}^{m=\text { Vacc }}=\eta(t) s_{0} \\
\Omega_{1}^{m=\text { Delta }}=\omega_{0} r_{0}\end{array}$ & NA \\
\hline
\end{tabular}


medRxiv preprint doi: https://doi.org/10.1101/2022.02.11.22270854; this version posted February 13, 2022. The copyright holder for this preprint

(which was not certified by peer review) is the author/funder, who has granted medRxiv a license to display the preprint in perpetuity.

It is made available under a CC-BY-NC-ND 4.0 International license .

\subsection{State-space transmission model for the Omicron variant and projection of Omicron spread from week 45 ,}

\section{1 to the end of the Omicron wave.}

For simplicity, we consider a hypothetical scenario where Omicron has successfully displaced all other circulating variants in South Africa and explore how the transmission dynamics of Omicron is shaped by the immune history of previously circulating strains and vaccination. Accordingly, at the time of writing, Omicron had replaced Delta in many countries that report variant-specific prevalence estimates, including South Africa. We do not consider variant co-circulation and the emergence of new variant during the Omicron wave, although such scenarios are certainly possible. Similar to the Delta variant, we consider a "Susceptible-Infectious-Recovered-Susceptible" model for Omicron that tracks infection history up to 3 repeat infections/immunizations, with additional Omicron-specific properties of immune evasion and enhanced transmissibility. The equations governing the model are as follows:

$$
\frac{d s_{0}}{d t}=-\lambda_{0} s_{0}+\mu-v s_{0}
$$

15

$$
\begin{aligned}
& \frac{d i_{0}}{d t}=\lambda_{0} s_{0}-\gamma_{0}^{\text {Omicron }} i_{0}-v i_{0} \\
& \frac{d r_{0}}{d t}=\gamma_{0}^{\text {Omicron }} i_{0}-\omega_{0} r_{0}-v r_{0}
\end{aligned}
$$

$$
\begin{aligned}
& \frac{d s_{1}^{m}}{d t}=-\lambda_{1}^{m} s_{1}^{m}+\Omega_{1}^{m}-v s_{1}^{m} \\
& \frac{d i_{1}^{m}}{d t}=\lambda_{1}^{m} s_{1}^{m}-\gamma_{1}^{m} i_{1}^{m}-v i_{1}^{m} \\
& \frac{d r_{1}^{m}}{d t}=\gamma_{1}^{m} i_{1}^{m}-\omega_{1} r_{1}^{m}-v r_{1}^{m}
\end{aligned}
$$

25

$$
\begin{aligned}
& \frac{d s_{2}^{n}}{d t}=-\lambda_{2}^{n} s_{2}^{n}+\Omega_{2}^{n}-v s_{2}^{n} \\
& \frac{d i_{2}^{n}}{d t}=\lambda_{2}^{n} s_{1}^{n}-\gamma_{2}^{n} i_{2}^{n}-v i_{1}^{n}
\end{aligned}
$$




$$
\frac{d r_{2}^{n}}{d t}=\gamma_{2}^{n} i_{2}^{n}-\Omega_{2}^{n}-v r_{2}^{n}
$$

With the following expression for the force-of-infection:

(1)

$$
\begin{gathered}
\lambda_{0}=\frac{R_{0}^{\text {Omicron }}}{R_{0}^{\text {Delta }}} \times \frac{\gamma_{0}^{\text {Omicron }}}{\gamma_{0}^{\text {Delta }}} \times \alpha_{\text {scaling }} \times \beta_{\text {Delta }} \times(1+\Delta \beta \cos (2 \pi(t+\phi) / T)) \times\left(i_{0}+\sum_{m \in M} \epsilon_{1}^{m} i_{1}^{m}+\sum_{n \in N} \epsilon_{2}^{n} i_{2}^{n}\right) \\
\lambda_{1}^{m}=\theta_{1}^{m} \lambda_{0} \\
\lambda_{2}^{n}=\theta_{2}^{n} \lambda_{0}
\end{gathered}
$$

10

The infectious periods for $i_{1}^{m}$ and $i_{2}^{n}$ can be expressed as follows with respect to $i_{0}$ 's:

$$
\begin{aligned}
& \frac{1}{\gamma_{1}^{m}}=\frac{\kappa_{1}^{m}}{\gamma_{0}^{\text {Omicron }}} \\
& \frac{1}{\gamma_{2}^{n}}=\frac{\kappa_{2}^{n}}{\gamma_{0}^{\text {Omicron }}}
\end{aligned}
$$

The definitions of the state variables are presented in Table S5, and the definitions of the model parameters are in Table S6.

Based on the transmission model, we explored how the degree of Omicron's immune evasion against infection $\sigma_{O m}^{i}$ 20 and onward transmission $\sigma_{O m}^{t \mid i}$ would shape the trajectory of the epidemic (See Section 5.2 and Table S2 for the definition of $\sigma_{O m}^{i}$ and $\sigma_{O m}^{t \mid i}$ ). We scanned through values of $\sigma_{O m}^{i}$ and $\sigma_{O m}^{t \mid i}$ ranging from 0 to 1 , with a step size of $1 / 30$. We also considered a potential change in the generation interval (GI) of Omicron when compared to the Delta variant. We explored possible values for Omicron's generation time, including 3, 4, 5, and 6 days. For each pair value pair of $\sigma_{O m}^{i}$ and $\sigma_{O m}^{t \mid i}$ and GI, we fit the ratio of basic reproduction number between Omicron and Delta $\frac{R_{0}^{O m i c r o n}}{R_{0}^{D e l t a}}$ so 25 that the growth rate of Omicron matched the observed initial growth of the Omicron wave (Figure S2). We fit the fraction of individual in the $i_{0}$ compartment at week 45, 2021 so that the peak of the projected incidence of Omicron infections matched the observed Omicron case incidence.

We then calculated the characteristics of the projected Omicron wave, including the estimated 1) infection attack rate, 2) epidemic duration, 3) fraction of reinfections/breakthrough infections among all infections, 4) the relative reduction of realized GI (average GI over both primary infections and reinfections/breakthrough of infections) with respect to intrinsic GI, and the 5) infection case ratio (number of cases reported to the Dr Kenneth Kaunda District during the Omicron wave divided by the total number of projected Omicron infections), for a given $\sigma_{O m}^{i}$ and $\sigma_{O m}^{t \mid i}$ and GI. Figure S4 visualizes projected characteristics of the Omicron wave as a function of $\sigma_{O m}^{i}$ and $\sigma_{O m}^{t \mid i}$ and GI. Figure 3A- 
E represents a scenario where the GI of Omicron is 4 days, shorter than Delta (8), where white dots represent our best knowledge of the degree of Omicron's evasion of prior immunity against infection and onward transmission $(11,17$, $18,24)$.

Table S5: State variables of the Omicron variant compartmental transmission model.

\begin{tabular}{|c|c|c|}
\hline Variable name & Variable type & Definition \\
\hline$p O m \in\{p O m\}$ & Categorical & $\begin{array}{l}\text { pOm } \in\{p O m\}=\{\text { D614G, Beta, Delta, Delta, Others, Vacc }\} \text { denotes immune history including only } \\
\text { one antigen exposure by pre-Omicron infection (including D614G, Beta, Delta, and other variants } \\
\text { other than Omicron ("Others") in South Africa) or a primary schedule of vaccination ("Vacc") }\end{array}$ \\
\hline$m \in M$ & Categorical & $\begin{array}{l}m \text { Indicates the type of primary SARS-CoV-2 antigen exposure from the set } M=\{p O m\} \cup\{O m\}, \\
\text { where and } O m \text { denotes immune history including only one antigen exposure to Omicron. }\end{array}$ \\
\hline$n \in N$ & Categorical & $\begin{array}{l}n \text { Indicates the specific combination of primary and secondary SARS-CoV-2 antigen exposures from } \\
\text { the set } N=\{p O m-p O m\} \cup\{p O m-O m\} \cup\{O m-O m\} \text {, where } p O m-p O m \text { denotes immune } \\
\text { history including at least two antigen exposures with first exposure being either pre-Omicron infection } \\
\text { or vaccination and second exposure either pre-Omicron infection or vaccination; } p O m-O m \text { denotes } \\
\text { immune history including at least two antigen exposures with first exposure being either pre-Omicron } \\
\text { infection or vaccination and second exposure being Omicron infection; } O m-O m \text { denotes immune } \\
\text { history including at least two antigen exposures with first and second exposure both being Omicron } \\
\text { infections. }\end{array}$ \\
\hline$s_{0}$ & State variable & $\begin{array}{l}\text { Fraction of susceptible in the population who are fully naïve against any SARS-CoV-2 infection and } \\
\text { are unvaccinated. }\end{array}$ \\
\hline$s_{1}^{m}$ & State variable & $\begin{array}{l}\text { Fraction of susceptible in the population who have experienced one infection or a full schedule of } \\
\text { vaccination, with } m \text { denoting the type of primary antigen exposure (i.e., variant type if infection or if } \\
\text { primed by vaccination). }\end{array}$ \\
\hline$s_{2}^{n}$ & State variable & $\begin{array}{l}\text { Fraction of susceptible in the population who have experienced two or more infections and } \\
\text { immunization combined, with } n \text { denoting the type of primary and secondary antigen exposure } \\
\text { (conferred by (re)infections or vaccinations). We assume that the first two immunizations will have the } \\
\text { strongest impact on the level of long-term protective immunity. }\end{array}$ \\
\hline$i_{0}$ & State variable & Fraction of population who got infected by Omicron from population in $s_{0}$ \\
\hline$i_{1}^{m}$ & State variable & Fraction of population who got infected by Omicron from population in $s_{1}^{m}$ \\
\hline$i_{2}^{n}$ & State variable & Fraction of population who got infected by Omicron from population in $s_{s}^{n}$ \\
\hline$r_{0}$ & State variable & $\begin{array}{l}\text { Fraction of population who recovers from Omicron in } i_{0} \text { compartment and enjoys a temporary period } \\
\text { of full immunity ( } 100 \% \text { protection) against any reinfection. }\end{array}$ \\
\hline$r_{1}^{m}$ & State variable & $\begin{array}{l}\text { Fraction of population who recovers from reinfection/breakthrough of Omicron infection in } i_{1}^{m} \\
\text { compartment and enjoys a temporary period of full immunity (100\% protection) against any } \\
\text { reinfection. }\end{array}$ \\
\hline$r_{2}^{n}$ & State variable & $\begin{array}{l}\text { Fraction of population who recovers from further reinfection/breakthrough of Omicron infection in the } \\
i_{2}^{n} \text { compartment and enjoys a temporary period of full immunity (100\% protection) against any } \\
\text { reinfection. }\end{array}$ \\
\hline
\end{tabular}

Table S6: Model parameters for the Omicron variant transmission model.

\begin{tabular}{|c|l|l|l|}
\hline Parameter & Definition & Value & Notes/Reference \\
\hline$\mu$ & Annual birth rate in South Africa & 0.026 year $^{-1}$ & \\
\hline
\end{tabular}




\begin{tabular}{|c|c|c|c|}
\hline$v$ & Annual death rate in South Africa & 0.008 year $^{-1}$ & \\
\hline$\beta_{\text {Delta }}$ & Baseline transmission rate of Delta & 1.02 day $^{-1}$ & See Table S4. \\
\hline$\Delta \beta$ & Magnitude of seasonal forcing & 0.15 & $(64)$ \\
\hline$\phi$ & $\begin{array}{l}\text { Phase of seasonal forcing in South } \\
\text { Africa }\end{array}$ & 180 days & $\begin{array}{l}\text { *South Africa is located in the Southern } \\
\text { Hemisphere; we assume a peak of seasonal } \\
\text { transmission during winter months }\end{array}$ \\
\hline$\theta_{1}^{m}$ & $\begin{array}{l}\text { Reduction in susceptibility to } \\
\text { Omicron infection due to protection } \\
\text { from primary antigen exposure. }\end{array}$ & $R R_{m}^{i}(\mathrm{Om})$ & $\begin{array}{l}\text { Taken into consideration of Omicron's } \\
\text { ability }\left(\sigma_{O m}^{i}\right) \text { to evade immunity conferred by } \\
\text { non-Omicron variant See Table S2 for } \\
\text { detailed definition for each term of } \\
R R_{m}^{i}(O m) \text { when } m \text { (Table S5) takes } \\
\text { different value. }\end{array}$ \\
\hline$\theta_{2}^{n}$ & $\begin{array}{l}\text { Reduction in susceptibility to } \\
\text { Omicron infection due to protection } \\
\text { from primary and secondary antigen } \\
\text { exposures. }\end{array}$ & $R R_{n}^{i}(\mathrm{Om})$ & $\begin{array}{l}\text { Taken into consideration of Omicron's } \\
\text { ability }\left(\sigma_{O m}^{i}\right) \text { to evade immunity conferred by } \\
\text { non-Omicron variant See Table S2 for } \\
\text { detailed definition for each term of } \\
R R_{n}^{i}(O m) \text { when } n \text { (Table S5) takes different } \\
\text { value. }\end{array}$ \\
\hline$\alpha_{\text {scaling }}$ & $\begin{array}{l}\text { Transmission scaling factor to } \\
\text { account for factors such as non- } \\
\text { pharmaceutical interventions or } \\
\text { heterogeneity in mixing patterns }\end{array}$ & 0.44 (Fitted) & $\begin{array}{l}\text { Estimated during the Delta period, and } \\
\text { assuming this has not change during the } \\
\text { period of Delta wave. See also Table S4 }\end{array}$ \\
\hline $1 / \gamma_{0}^{\text {Delta }}$ & $\begin{array}{l}\text { Infectious period of } \\
\text { Delta's primary infection } i_{0}\end{array}$ & 5 days & See Table S4. \\
\hline $1 / \gamma_{0}^{\text {Omicron }}$ & $\begin{array}{l}\text { Infectious period of Omicron's } \\
\text { primary infection } i_{0}\end{array}$ & $3,4,5,6$ days & $\begin{array}{l}4 \text { days (shorter than Delta) for the reference } \\
\text { scenario }(21) \text {, while sensitivity analysis of 3- } \\
6 \text { days to explore the deviation of Omicron's } \\
\text { infectious period deviating from Delta. }\end{array}$ \\
\hline$\frac{R_{0}^{\text {Omicron }}}{R_{0}^{\text {Delta }}}$ & $\begin{array}{l}\text { Ratio between Omicron and Delta's } \\
\text { reproduction number }\end{array}$ & Free parameter & $\begin{array}{l}\text { Free parameter to be explored along with } \\
\text { degree of immune evasion from Omicron } \\
\text { against susceptibility } \sigma_{O m}^{i} \text { and transmission } \\
\sigma_{O m}^{t \mid i} \text { (see Table S2). }\end{array}$ \\
\hline$\kappa_{1}^{m}$ & $\begin{array}{l}\text { Reduction in terms of duration of } \\
\text { shedding due to protection from } \\
\text { primary antigen exposure }\end{array}$ & $\left(R R_{m}^{t \mid i}(\mathrm{Om})\right)^{\frac{1}{2}}$ & $\begin{array}{l}\text { Assuming that reduction of onward } \\
\text { transmission (Table S2) is evenly split } \\
\text { between reduction in duration and intensity } \\
\text { of shedding. See Table } \mathrm{S} 2 \text { for detailed } \\
\text { definition for each term of } R R_{m}^{t \mid i}(\mathrm{Om}) \text { when } \\
m \text { (Table S5) takes different value. }\end{array}$ \\
\hline $1 / \gamma_{1}^{m}$ & $\begin{array}{l}\text { Infectious period of Omicron's first } \\
\text { reinfection/breakthrough of infection } \\
i_{1}^{m}\end{array}$ & $\kappa_{1}^{m} / \gamma_{0}$ & NA \\
\hline$\kappa_{2}^{n}$ & $\begin{array}{l}\text { Reduction in terms of duration of } \\
\text { shedding due to protection from } \\
\text { primary and secondary antigen } \\
\text { exposures }\end{array}$ & $\left(R R_{n}^{t \mid i}(O m)\right)^{\frac{1}{2}}$ & $\begin{array}{l}\text { Assuming that reduction of onward } \\
\text { transmission (Table S2) is evenly split } \\
\text { between reduction in duration and intensity } \\
\text { of shedding. See Table S2 for detailed }\end{array}$ \\
\hline
\end{tabular}




\begin{tabular}{|c|c|c|c|}
\hline & & & $\begin{array}{l}\text { definition for each term of } R R_{n}^{t \mid i}(\mathrm{Om}) \text { when } \\
n \text { (Table S5) takes different value. }\end{array}$ \\
\hline $1 / \gamma_{2}^{n}$ & $\begin{array}{l}\text { Infectious period of Omicron's } \\
\text { second reinfection/breakthrough } \\
\text { infection } i_{2}^{n}\end{array}$ & $\kappa_{2}^{n} / \gamma_{0}$ & NA \\
\hline$\epsilon_{1}^{m}$ & $\begin{array}{l}\text { Reduction in terms of intensity of } \\
\text { shedding due to protection from } \\
\text { primary antigen exposures }\end{array}$ & $\left(R R_{m}^{t \mid i}(O m)\right)^{\frac{1}{2}}$ & $\begin{array}{l}\text { Assuming that reduction of onward } \\
\text { transmission (Table S2) is evenly split } \\
\text { between reduction in duration and intensity } \\
\text { of shedding. See Table } \mathrm{S} 2 \text { for detailed } \\
\text { definition for each term of } R R_{m}^{t \mid i}(\mathrm{Om}) \text { when } \\
m \text { (Table S5) takes different value. }\end{array}$ \\
\hline$\epsilon_{2}^{n}$ & $\begin{array}{l}\text { Reduction in terms of intensity of } \\
\text { shedding due to protection from } \\
\text { primary and secondary antigen } \\
\text { exposures }\end{array}$ & $\left(R R_{n}^{t \mid i}(\mathrm{Om})\right)^{\frac{1}{2}}$ & $\begin{array}{l}\text { Assuming that reduction of onward } \\
\text { transmission (Table S2) is evenly split } \\
\text { between reduction in duration and intensity } \\
\text { of shedding. See Table } \mathrm{S} 2 \text { for detailed } \\
\text { definition for each term of } R R_{n}^{t \mid i}(\mathrm{Om}) \text { when } \\
n \text { (Table S5) takes different value. }\end{array}$ \\
\hline $1 / \omega_{0}$ & $\begin{array}{l}\text { Duration of period of full immunity } \\
\text { following primary infection with } \\
\text { Omicron }\end{array}$ & 60 days & Assumed \\
\hline $1 / \omega_{1}$ & $\begin{array}{l}\text { Duration of period of full immunity } \\
\text { following primary infection with } \\
\text { Omicron following first reinfection } \\
\text { with Omicron }\end{array}$ & 60 days & Assumed \\
\hline $1 / \omega_{2}$ & $\begin{array}{l}\text { Duration of period of full immunity } \\
\text { following primary infection with } \\
\text { Omicron following repeat } \\
\text { reinfections with Omicron }\end{array}$ & 60 days & Assumed \\
\hline$\Omega_{1}^{m}$ & $\begin{array}{l}\text { Operator that maps the primary } \\
\text { exposure (Delta } \\
\text { infection/vaccination) to different } \\
\text { susceptible compartment } s_{1}^{m} \\
\text { following the primary infection. }\end{array}$ & $\begin{array}{l}\Omega_{1}^{m=\text { omicron }}=\omega_{0} r_{0} \\
\Omega_{1}^{m \neq \text { omicron }}=0\end{array}$ & NA \\
\hline$\Omega_{2}^{n}$ & $\begin{array}{l}\text { Operator that maps the circulating } \\
\text { variant of interest to the imprinted } \\
\text { susceptible compartment } \\
s_{2}^{n} \text { following the first reinfection }\end{array}$ & $\begin{array}{l}\Omega_{2}^{n=p O m-p O m}=0 \\
\Omega_{2}^{n \neq p O m-p O m}=\omega_{1} r_{1}^{m-O m}\end{array}$ & NA \\
\hline
\end{tabular}

\section{Modelling the transmission dynamics of Omicron, Delta and a hypothetical variant $X$ after the Omicron} wave

Here we modify the transmission model described in Section 5 to evaluate the possibility of a fifth epidemic wave after the Omicron wave. Specifically, we evaluate the potential recurrence of three variants independently: Omicron, Delta, and a hypothetical variant $\mathrm{X}$, where $\mathrm{X}$ is at equal antigenic distance from Omicron and Delta. We consider the projected Omicron wave for the reference scenario shown in Figure 3F. After the Omicron wave, two new population 
groups need to be taken into consideration based on their antigen exposure(s): 1) individuals who were primed by the Omicron variant, which accounts for $23 \%$ of the population; and 2 ) individuals who were primed by a non-Omicron variant (through infection or vaccination) then reinfected by Omicron, which accounts for $46 \%$ of the population. For any given variant of interest, we denote the level of protection (against infection) conferred after primary Omicron infection as $1-R R_{O m}^{i}$ (variant of interest) and after Omicron reinfection/vaccine breakthrough as $1-$ $R R_{n O m-O m}^{i}$ (variant of interest), where as $R R^{i}$ stands for the relative risk of acquiring infection when compared to an immunologically naïve individual $\left(1-R R^{i}=0 \%\right.$ indicates no protection while $1-R R^{i}=100 \%$ indicates perfect protection). For simplicity, we assume protection against transmission $1-R R^{t \mid i}$ remains constant at $60 \%$ ( 18 , 24).

\subsection{Omicron transmission model}

For the Omicron transmission model, we first consider the same parameters as in the reference scenario as shown in Figure $3 \mathrm{~F}$, with generation time of 4 days, $\sigma_{O m}^{i}=0.7, \sigma_{O m}^{t \mid i}=0.2$, and the estimated $R_{0}^{\text {Omicron }} / R_{0}^{\text {Delta }}=2.37$. However, for the two population groups of interest 1) those who were primed by Omicron 2) those who have experienced a Omicron reinfection/breakthrough we consider their relative risk (with respect to naïve population) of acquiring Omicron infection as $R R_{O m}^{i}$ (Omicron) and $R R_{n O m-O m}^{i}$ (Omicron), respectively. In Figure 3G, we use the transmission model to evaluate the growth rate of Omicron when Omicron is reintroduced into the population for all combinations of $R_{O m}^{i}$ (Omicron) and $R R_{n O m-O m}^{i}$ (Omicron) ranging from 0 to 1 . A growth rate larger than 0 indicates that the Omicron variant is above the epidemic threshold, leading to a recurring fifth epidemic wave, while a growth rate lower than 0 indicates that the Omicron variant will not trigger another outbreak after the fourth wave. We further consider a scenario (Figure $3 \mathrm{H}$ ) where the contact rate is twice that of the one during the fourth wave, i.e. $\alpha_{\text {scaling }}=0.44 \times 2=0.88($ Table S6).

\subsection{Delta transmission model}

To evaluate the risk of Delta recurrence, we consider the same Delta transmission model described in Materials and Methods Section 5.5. However, for the population groups 1) who were primed by Omicron 2) who have experienced a Omicron reinfection/vaccine breakthrough, we consider their relative risk (with respect to naïve individuals) of acquiring Delta infection as $R R_{O m}^{i}\left(\right.$ Delta) and $R R_{n O m-O m}^{i}($ Delta), respectively. In Figure 3I, we use the transmission model to evaluate the growth rate of the Delta variant when it is reintroduced into the population for any combination of $R_{O m}^{i}$ (Delta) and $R R_{\text {nOm-Om }}^{i}$ (Delta) ranging from 0 to 1 . We further consider a scenario (Figure 3J) where the contact rate is twice that of the one during the fourth wave, i.e., $\alpha_{\text {scaling }}=0.44 \times 2=0.88$ (Table S4).

\subsection{Transmission model of the hypothetical variant $X$}

For the transmission model of a hypothetical new variant $\mathrm{X}$, we consider that variant $\mathrm{X}$ has the same basic reproduction number and generation time as the Delta variant, and the contact rate is twice that of the fourth wave, i.e., $\alpha_{\text {scaling }}=$ $0.44 \times 2=0.88$ (Table S4). We additionally assume that variant $\mathrm{X}$ is antigenically equally distinct from both Omicron and pre-Omicron variant so that the relative risks of reinfection are equal irrespective of the primed strain i.e., $R R_{O m}^{i}(X)=R R_{n O m}^{i}(X)$. The rest of the model is the same as for Delta. 


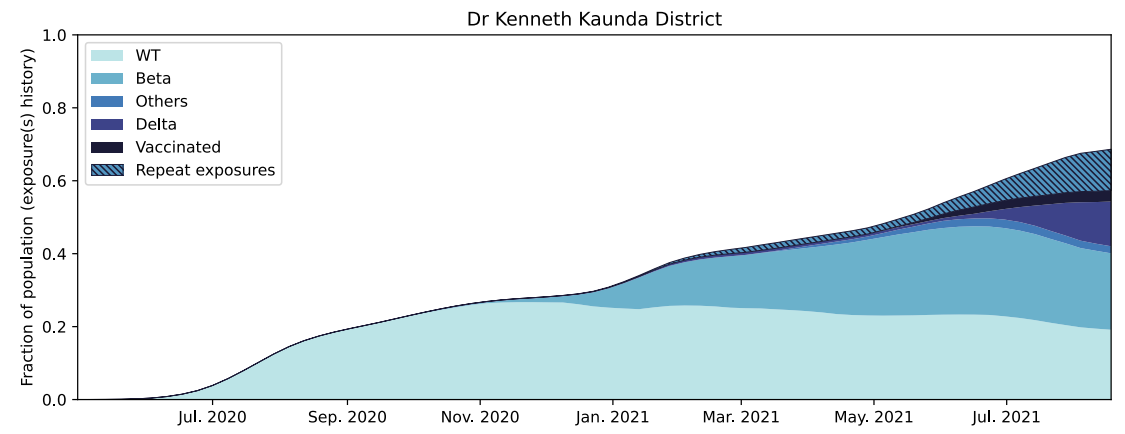

Fig. S1: SARS-CoV-2 antigen exposure history by the end of PHIRST-C (September 2021) at the District of Dr Kenneth Kaunda.

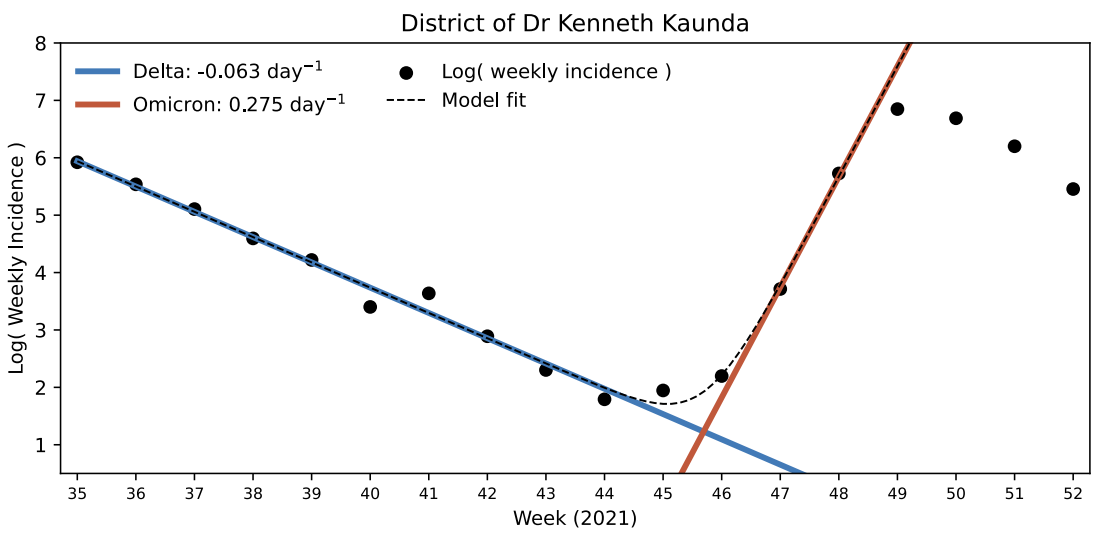

Fig. S2: Estimated case growth rate of Delta and Omicron between weeks 35 and 52 of 2021. Dots are the logarithmic of the weekly incidence of SARS-CoV-2 cases reported to the District of Dr Kenneth Kaunda between weeks 35 and 52 of 2021. The blue line is the fitted exponential decaying of Delta Incidence while the red line is the fitted exponential growth of Omicron wave and the dashed line is the fitted convolution of the two variants.

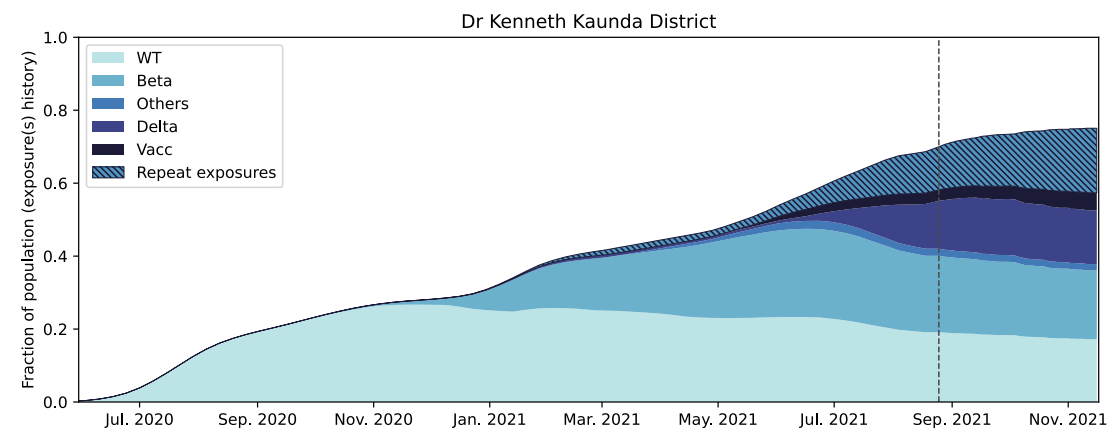

Fig. S3: Projection of SARS-CoV-2 antigen exposure history from the end of PHIRST-C (week 35 of 2021, dashed line) and until the emergence of Omicron (week 45 of 2021, Figure S2) at the District of Dr Kenneth Kaunda. 
medRxiv preprint doi: https://doi.org/10.1101/2022.02.11.22270854; this version posted February 13, 2022. The copyright holder for this preprint (which was not certified by peer review) is the author/funder, who has granted medRxiv a license to display the preprint in perpetuity.

It is made available under a CC-BY-NC-ND 4.0 International license .
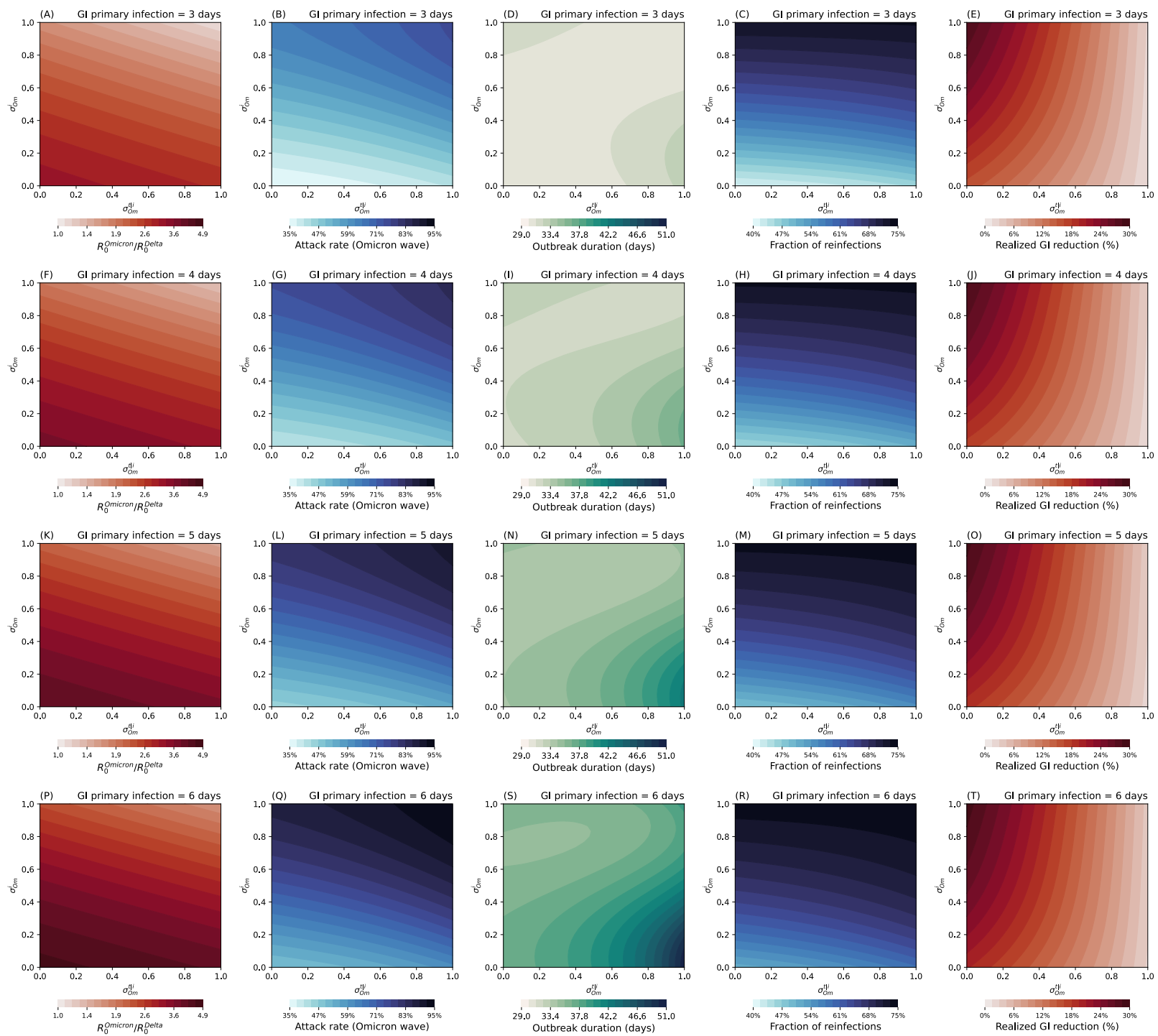

Fig. S4: The characteristics of the Omicron epidemic wave as a function of $\sigma_{O m}^{i}$ and $\sigma_{O m}^{t \mid i}$ and generation time.

Each row corresponds to different values of generation time (GI). Column one corresponds to ratio of basic reproduction number between Omicron and Delta. Column two corresponds to infection attack rate. Column three corresponds to duration of the epidemic. Column four corresponds to fraction of reinfections/breakthrough of infections among all infections. Column five corresponds to the relative reduction of realized GI (average GI over both primary infections and reinfections/breakthrough infections) with respect to the intrinsic GI. 